\title{
Human health risk assessment of heavy metals in soils and commonly consumed food crops from quarry sites located at Isiagwu, Ebonyi State
}

\author{
Uchechi Bliss ONYEDIKACHI, ${ }^{*}$ Donatus Chuka BELONWU, ${ }^{2}$ and Mattew Owhonda WEGWU ${ }^{2}$ \\ ${ }^{1}$ Department of Biochemistry, College of Natural Sciences, Michael Okpara University of Agriculture, Umudike, \\ Umuahia, Abia State, Nigeria \\ ${ }^{2}$ Department of Biochemistry, Faculty of Chemical Science, University of Port Harcourt, River State, Nigeria
}

\begin{abstract}
In view of ensuring healthy agricultural foods for human consumption, this study assessed the human health risk implicated in selected heavy metals in some commonly consumed vegetables, tubers, nuts and fruits grown around the quarry sites at Ishiagu, Ebonyi State, Nigeria. Samples from agriculture area of Umudike, Abia State, Nigeria, constituted the control. The concentration of $\mathrm{Mn}, \mathrm{Zn}, \mathrm{Fe}, \mathrm{Cu}, \mathrm{Cd}$, and $\mathrm{Pb}$, were determined using atomic absorption spectrometry. The potential non-carcinogenic health risk for consumers which included Estimated Daily Intake (EDI) and Target Hazard Quotients (THQ) for $\mathrm{Pb}, \mathrm{Fe}, \mathrm{Mn}, \mathrm{Zn}, \mathrm{Cd}$ and $\mathrm{Cu}$ while carcinogenic health risk using Cancer Slope Factors (CSF) was established for $\mathrm{Cd}$ and $\mathrm{Pb}$. Relative abundance of heavy metals across the locations and all samples was in the order $\mathrm{Fe}>\mathrm{Mn}>\mathrm{Zn}>\mathrm{Pb}>\mathrm{Cu}>\mathrm{Cd}$. There was statistical significant effect of quarrying activities on the concentration of the heavy metals ( $\mathrm{Fe}, \mathrm{Mn}, \mathrm{Zn}, \mathrm{Pb}, \mathrm{Cu}$, Cd) at $p<0.05$ level. Based on the observed bioconcentration factors, cassava showed more hyperaccumulation potential compared to other samples. Pumpkin and bitter leaf also could be used in remediation owing to their high bioaccumulation index for $\mathrm{Pb}$ and $\mathrm{Zn}$. THQ obtained for $\mathrm{Mn}$ and $\mathrm{Pb}$ were $>1$ indicating that the residents at the quarry site may be exposed to potential non-carcinogenic health risk due to $\mathrm{Mn}$ and $\mathrm{Pb}$ intoxication. With respect to US EPA prescriptions, average carcinogenic risk values obtained for $\mathrm{Pb}$ and $\mathrm{Cd}$ in this study indicated a lifetime (70 years) probability of contracting cancer suggesting that they be placed for further consideration as chemicals of concern with respect to the assessed locals.
\end{abstract}

Keywords: health risk assessment, bioconcentration factors, daily intake of heavy metals, target hazard quotient, carcinogenic risk, heavy metal.

\section{Introduction}

Health risks assessment has to do with evaluating the risk to a particular organism or population following exposure to certain chemical agent(s) by taking into consideration the characteristics of the agent and the target system it affects. The model for health risk assessment begins with formulation of a problem followed by identification of hazard, characterization of hazard, assessment of exposure and then risk characterization [1]. The public are becoming progressively concerned and conscious of the presence of toxic chemicals in the environment that pose a threat to human health. Even though, some persons think these concerns are over exaggerated and unnecessary, the awareness of the effects of these contaminants in our food, drinking water and air has increased over the years. Health risk data can assist the government and regulatory bodies in ascertaining and abating possible environmental hazards. Also, using these data the populace with exposures to hazards can work effectively with decision makers in ameliorating environmental pollution by learning more about the hazard and assisting during the implementation of environmental remediation programs [1].

Food crops like vegetables, tubers, fruits and nuts cultivated in contaminated lands can amass toxic heavy metals. The soil in these areas is the principal source of these toxicants. Human exposure to toxic heavy metals is via inhalation of dust, intake of contaminated water and food crops. Heavy metals via food accumulate in the several organs causing altered metabolism in liver and kidney, cardiovascular, nervous and bone disorders [2].

Extractive industries like quarries play vital economic roles in countries like Nigeria through the provision of employment and contribution to gross domestic product. Quarrying is a major means of diversifying the economy of many African countries and is a predominant economic activity in some Nigerian states like Ebonyi State. Quarrying business is a means of generating internal income for most local governments in Ebonyi State. Economic products like limestone, marble, sandstone, slate etc. used in building and construction are the major

*Corresponding author. E-mail address: ucheonyedi@gmail.com; ub.onyedikachi@mouau.edu.ng (Uchechi Onyedikachi). 
products of quarrying and stone crushing industries like crush rock. Currently, there are over 130 small and medium scale quarrying industries employing over 3,000 workers in the state. The abundance of sedimentary rocks and increased demand for ceramics, building materials and granite has led to increased quarry sites, quarrying activities and consequent environmental pollution. Like other anthropogenic activities, quarrying significantly impact the environment and poses risk to human health both to workers at the mines and families who live around these areas [3].

Most farmlands in Ishiagu are situated close to the mining sites resulting in increased uptake of heavy metals by crops. This result in not only observable morphological and physiological changes in crops but also, increased risk of human intoxication [4]. It is therefore imperative to evaluate the concentration of heavy metals like lead, zinc, cadmium, iron etc. in edible plant tissues, since they are possible toxicological hazards from such site. This is the focus of present research.

Environmental pollution is a global challenge with enormous impact on human health. Ecological pollution is prevalent in densely populated and high industrialized metropolis. Human exposure to environmental contaminants has increased over the years thus the increase in global concerns on the effects of pollutants on human health. Some industries in the western world now adopt strategies to avoid environmental pollution as an integral aspect of their general plans [5].

Environmental pollution constitutes one of the most unsafe occurrences resulting from human activities and natural events. Metal mining, processing and smelting are common human activities with negative impact on the environment contributing to environmental pollution. High concentrations of heavy metals and metalloids accumulate at mine sites following the extraction of elements with economic value. This results to contamination of such environs with metals like As, $\mathrm{Cd}, \mathrm{Cr}, \mathrm{Pb}, \mathrm{Ni}, \mathrm{Sb}$ and $\mathrm{Zn}$. Several studies have been conducted on heavy metal contamination of areas around mines. These studies are centered on methods for assessing the factors controlling the distribution, behavior and environmental impact of these contaminants with regards to both plant and human health [5].

The mining and processing of minerals release crushed wastes and tailings which can pose possible threats to the environment when exposed to further weathering. Anthropogenic pollution may result following the dispersal of these contaminants into the environment around mine by wind, water and other agents of denudation [6].

Heavy metals that find their way into the environment may be geo-accumulated or bioaccumulated thus it becomes important to understand the sources of heavy metals, the effect of their accumulation in soil, water and on plant system for risk assessment. Soils, food crops and sea food gotten from vicinities with close proximity to mine sites possess an abnormal elevated level of heavy metals and polycyclic aromatic hydrocarbons. The effect of environmental contamination will be more pronounced in areas with dense population [6]. Ingestion of food crops contaminated with heavy metals decreases the bioavailability of some essential nutrients. This can deplete the immunological response leading to gastrointestinal cancer, intrauterine growth retardation, impaired psychosocial facilities, etc. [7].

Polluted areas including lands and aquatic habitats can be cleaned up through the use of plants in a process known as phytoremediation. Such plants should have high translocation potentials and quick biomass production. This new technique for restoring polluted areas has received substantial consideration in recent times [8].

Despite the negative impact environmental pollution, the Nigerian government have not paid serious attention to environmental pollution and degradation [9]. An efficient environmental remediation will be dependent on assessment of the health risk considering several age groups and the joint effort of both government, regulatory bodies and voluntary service [5].

Environmental implications of quarrying activities. Rock and rock mineral exploitation activities are sources of additional heavy metals in soils. Blasting, crushing of rocks and use of explosives and heat to produce granite chips release particulate matter and dust of different metallic constituents from the machineries and blasting processes. Heavy metals are non-biodegradable, difficult to remove and on reaching the soil would accumulate by bonding to clay and organic colloids [10]. Extractive industries like quarries have been implicated as a major source of exposure to occupational health hazard. Rock blasting with explosives results in air pollution, noise pollution and significant damage to the biosphere. Air pollution resulting from quarry sites constitutes a menace, affecting human and plant health. Damage to internal structures, chemical effect and leaf abrasion are the long term effects of dusty air that affect plant survival. Quarrying generates particles of different chemical composition in the form of smoke, dust and vapor which could remain suspended in air for long and transported from area of generation to far distant places. Inhaling such air results in respiratory problems like pneumoconiosis [3]. Like other mining operations, quarrying is a destructive anthropogenic activity whose socio-economic benefits may be unable to compensate for the overall detrimental effects on natural ecosystems. Compaction by heavy machinery produces various impacts on the air, water, soil, earth surface, flora and fauna, and humans [5]. 
Area of study. Samples were collected from compounds and farmlands within the industrialized area of Ishiagu located in Ivo local government area of Ebonyi State, Nigeria. This is located within latitude $5^{0} 52^{\prime}$ to $5^{0} 60^{\prime} \mathrm{N}$ and longitudes $7^{0} 30^{\prime}$ to $7^{0}$ 37 " E. Geographically, Ishiagu is part of the Cross River Basin and is endowed with mineral resources like ores (lead and zinc). Owing to increased anthropological activities, this area which was originally situated within the tropical rain forest is gradually been replaced by forest Savanna [4].

Mining, quarrying and farming are the major means of livelihood in this area. The major crops grown are cassava, rice, yam, cocoyam, vegetables, nuts and fruits. Anthropogenic pollutants are released to adjacent lands including farms as a result of quarry activities and mineral exploitation in this area. As a result, the populace may be exposed to occupational health risk.

\section{Experimental}

\subsection{Materials}

The high purity reagents of analytical grades were obtained from BDH Chemicals Ltd., UK.

\subsection{Sample collection}

Freshly harvested vegetables (Telferia Occidentalis, Vernonia amygdalina, Talinum triangulare), tubers (Manihot esculenta, Dioscorea rodundata, Colocacia antiquorm), fruits (Citrus sinensis, Chrysophyllum albidun, Carica papaya), nuts (Cocos nucifera, Cola acuminata, Elaies guineensis) and soil samples were collected from farms around the industrial area. Soil samples were collected around the rhizosphere of each plant i.e., about $15-20 \mathrm{~cm}$ deep. The materials used for sample collection are: soil auger, kitchen knife, $60 \mathrm{~cm}$ meter rule, hoe and cutlass. In a bid to avoid cross-contamination each sample was sealed separately and transferred to the laboratory in less than twenty four hours.

\subsection{Food sample preparation/pretreatment}

Samples were peeled/shelled, washed three times with distilled water and allowed to dry at room temperature. Edible portions of food samples were oven dried to constant weight at $65{ }^{\circ} \mathrm{C}$ for 48 hours using Memmert drying oven. The dried samples were homogenized (ground to powder) using laboratory ceramic mortar and pestle. The homogenized samples were sieved with $2 \mathrm{~mm}$ sieve. The soil samples were also sieved to obtain fine particle size for easy digestion.

\subsection{Soil sample preparation and analysis}

Soil samples were air dried, crushed and sieved ( $2 \mathrm{~mm}$ screen). For heavy metals, $5 \mathrm{~g}$ of each sample was weighed into a clean, dry silica dish, covered and ignited in a furnace for $6 \mathrm{~h}$ at $500{ }^{\circ} \mathrm{C}$ until a grey white ash was obtained. The cover of the dish was opened to allow for escape of gases. To cool ash samples, $5 \mathrm{ml}$ of $10 \% \mathrm{HCl}$ was added to enhance dissolution and $5 \mathrm{ml}$ of $10 \% \quad \mathrm{HNO}_{3}$ was added thereafter and set on a water bath to dissolve completely. The solution was transferred into a clean dry $50 \mathrm{ml}$ standard volumetric flask. The filtrate was made up to the $100 \mathrm{ml}$ mark with distilled water. The samples were accurately labeled and set for atomic absorption spectrometry (AAS) [11].

\subsection{Atomic absorption spectrometry}

Each of the metals concentrations in the filtrate were determined by atomic absorption spectrometry (ContrAA 300, Analytik Jena, Germany). It was calibrated using standard of the metals, which are given below: $\mathrm{Cd}(\lambda)=228.80 \mathrm{~nm}, \mathrm{~Pb}(\lambda)=283.30$ $\mathrm{nm}, \mathrm{Zn}(\lambda)=213.9 \mathrm{~nm}, \mathrm{Mn}(\lambda)=279.50 \mathrm{~nm}$, and Fe $(\lambda)=248.3 \mathrm{~nm}$. Zn and Mn were analyzed using a hollow cathode lamp (HCL) in a flame atomizer AAS, while $\mathrm{Cd}$ and $\mathrm{Pb}$ were analyzed using electrodeless discharge lamp (EDL) in the flame atomizer AAS [11]. The blank reagent and standard reference soil materials were included in each sample batch to verify the accuracy and precision of the digestion procedure. The extract was aspirated directly into the atomic absorption spectrometer machine. The carrier gas was acetylene and air: $70 \Psi$ [11]. Data were validated by checking for completeness, holding times, calibrations, specific blank analysis, and detection limits [12].

\subsection{Statistical analysis}

Mean metal concentrations were statistically treated with SPSS version 21.0. One way between subjects ANOVA (analysis of variance) was used to determine if there was a statistical significant difference in mean concentration of each heavy metal within and between groups. Also, turkey Post Hoc test or LSD was used to determine where this difference lies. Other statistical analyses and calculations were performed with Microsoft Excel.

\subsection{Health risk assessment}

The health risks associated with the consumption of heavy-metal-contaminated food crops were assessed based on the Estimated Daily Intake of Metals (EDIM) of heavy metals, Target Hazard Quotient (THQ), and Carcinogenic Risk (CR). The calculations were based on the standard assumption for integrate US EPA risk analysis, FDA, and USDOE standard as indicated in Table 1.

Estimated daily intake of heavy metals. The estimated daily intake of heavy metals was calculated based on the equation below:

$$
E D I M=\frac{\text { CHM } \times \text { Cf } \times \text { DFI }}{\text { Average Body weight }}
$$

where CHM, Cf and DFI represent the concentration of heavy metals, conversion factor and daily food intake respectively. A conversion factor of 0.085 was used to convert the fresh sample weight of to dry weight [6]. Calculations were made with reference to 
the standard assumption for an integrate US EPA risk analysis, considering adults and children with average body weights of $60 \mathrm{~kg}$ and $32.7 \mathrm{~kg}$ respectively. Average daily consumption of vegetables, fruits and nuts for adults and children was considered to be $0.345 \mathrm{~kg}$ person ${ }^{-1} \mathrm{day}^{-1}$ and $0.173 \mathrm{~kg}$ person $^{-1}$ day $^{-1}$ respectively. Average daily consumption of tubers for adults and children was considered to be $0.418 \mathrm{~kg}$ person ${ }^{-1} \mathrm{day}^{-1}$ and $0.209 \mathrm{~kg}$ person $^{-1}$ day $^{-1}$ respectively $[5,6,13]$.

Target hazard quotient /health risk index (HI). With regards to its recognition as a useful parameter in estimating health risk, the target hazard quotient/health risk index was used to give estimates of the non-carcinogenic health risk due to oral exposure to heavy metals. It is defined as the ratio between exposure (EDIM) and reference oral dose (RFD). RFD is defined as the daily oral exposure to a substance that will not result in any deleterious effect in a life time for a given human population. RFD is a useful tool in EPA's non-carcinogenic health risk assessment [11, 14, 15].

$$
T H Q=\frac{C H M * \text { Cfactor } * D F I}{R F D * A B W}=\frac{E D I M}{R f D}
$$

where THQ, EDIM, RFD and ABW represent the target hazard quotient, the estimated daily intake of heavy metals, reference oral dose and average body weights. Values for DFI, C factor and ABW remain the same as defined above.

If $\mathrm{THQ} \leq 1$, the studied population is considered to be safe.
If THQ $>1$ the populace are exposed to toxic levels of the substance (heavy metals).

Carcinogenic risk and ingestion cancer slope factors (CSFing). As described by US EPA [16] and ATSDR [17], ingestion cancer slope factors are used to evaluate the lifetime (average of 70 years) probability of contracting cancer due to certain levels of exposure to a potential carcinogen $[16,17]$. The carcinogenic risk is expressed as:

\section{$\mathrm{CR}=$ EDIM X CSFing}

where CR, EDIM and CSFing are the carcinogenic risk, estimated daily intake of heavy metals and ingestion cancer slope factor respectively. Carcinogenic risk index between $10^{-6}$ ( 1 in $1,000,000)$ and $10^{-4}(1$ in 10,000) indicates an interval of allowable predicted lifetime risks for cancer a causing agents. Thus, chemicals having risk factors less than $10^{-6}$ may not be treated as chemicals for further concern [11, 18-20].

Table 1. Toxicological characteristics of the investigated metals [11]

\begin{tabular}{lcc}
\hline Metals & $\begin{array}{c}\text { Ingestion } \\
\text { Reference Dose } \\
(\mathrm{mg} / \mathrm{kg} / \text { day })\end{array}$ & $\begin{array}{c}\text { Ingestion Cancer } \\
\text { Slope Factor } \\
(\mathrm{mg} / \mathrm{kg} / \text { day })\end{array}$ \\
\hline Iron & 0.007 & 0 \\
Chromium & 0.003 & 0.5 \\
cadmium & 0.001 & 0.38 \\
Lead & 0.0035 & 0.0085 \\
Zinc & 0.3 & 0 \\
Copper & 0.04 & 0 \\
\hline
\end{tabular}

Table 2. Permissible limit of heavy metal concentration in soil and plants

\begin{tabular}{|c|c|c|c|c|c|c|c|c|c|}
\hline \multirow[b]{2}{*}{ Metal } & \multirow[b]{2}{*}{ Germany } & \multicolumn{5}{|c|}{$\begin{array}{l}\text { Allowable limit of heavy metal concentration in soil [21] } \\
\qquad\left[\mu \mathrm{g} \mathrm{g}^{-1}\right]\end{array}$} & \multirow[b]{2}{*}{$\begin{array}{l}\text { Soil ref. } \\
\text { range }\end{array}$} & \multicolumn{2}{|c|}{$\begin{array}{l}\text { Allowable limit by FAO/WHO } \\
\text { [22] }\end{array}$} \\
\hline & & Netherlands & Sweden & USA & Ireland & $\begin{array}{l}\text { Dutch } \\
\text { Standard }\end{array}$ & & $\begin{array}{l}\text { FAO/WHO } \\
\text { edible plant }\end{array}$ & $\begin{array}{l}\mathrm{FAO} / \mathrm{WHO} \\
\text { medicinal plant }\end{array}$ \\
\hline $\mathrm{Cd}$ & 1.0 & 0.5 & 0.4 & 1.9 & 1.0 & 0.8 & $0.4-1.9$ & 0.21 & 0.3 \\
\hline $\mathrm{Cu}$ & 40 & 40 & 40 & 75 & 50 & 36 & $36-75$ & 3.0 & - \\
\hline $\mathrm{Fe}$ & - & - & - & - & - & - & - & 20 & - \\
\hline $\mathrm{Pb}$ & 70 & 40 & 40 & 15 & 50 & 85 & $15-85$ & 0.43 & 10.0 \\
\hline $\mathrm{Zn}$ & 150 & 100 & $100-150$ & 140 & 150 & 50 & $50-150$ & 47.4 & - \\
\hline $\mathrm{Mn}$ & - & - & - & - & - & - & 20-10E04 & 2.0 & - \\
\hline
\end{tabular}

\section{Results and discussion}

\subsection{Results}

Table 3. Mean concentration of heavy metals ( $\mathrm{mg} / \mathrm{kg}$ dry weight) in selected food crops from quarry site (Ishiagu) and residential area (Umudike)

\begin{tabular}{|c|c|c|c|c|c|c|}
\hline Heavy metals & Mn & Cd & Zn & $\mathbf{F e}$ & $\mathbf{P b}$ & $\mathbf{C u}$ \\
\hline \multicolumn{7}{|c|}{ Manihot esculenta } \\
\hline Q. Sample & $118.31 \pm 0.01^{\mathrm{b}}$ & $0.23 \pm 0.03^{c}$ & $4.10 \pm 0.10^{\mathrm{b}}$ & $2654.11 \pm 0.11^{\mathrm{c}}$ & $0.91 \pm 0.01^{\mathrm{c}}$ & $\mathrm{BDL} \pm 0.00^{\mathrm{a}}$ \\
\hline C. Sample & $245.90 \pm 0.00^{\mathrm{c}}$ & $\mathrm{BDL} \pm 0.00^{\mathrm{b}}$ & $6.10 \pm 0.00^{c}$ & $1496.40 \pm 0.00^{\mathrm{b}}$ & $\mathrm{BDL} \pm 0.00^{\mathrm{b}}$ & $\mathrm{BDL} \pm 0.00^{\mathrm{a}}$ \\
\hline \multicolumn{7}{|c|}{ Dioscorea rodundata } \\
\hline Q. Sample & $34.10 \pm 0.10^{c}$ & $\mathrm{BDL} \pm 0.00^{\mathrm{b}}$ & $3.23 \pm 0.03^{\mathrm{b}}$ & $264.10 \pm 0.10^{c}$ & $\mathrm{BDL} \pm 0.00^{\mathrm{a}}$ & $\mathrm{BDL} \pm 0.00^{\mathrm{a}}$ \\
\hline
\end{tabular}


Onyedikachi et al. / Ovidius University Annals of Chemistry 29 (2018) 8 - 24

\begin{tabular}{|c|c|c|c|c|c|c|}
\hline Heavy metals & Mn & Cd & $\mathbf{Z n}$ & $\mathbf{F e}$ & $\mathbf{P b}$ & $\mathbf{C u}$ \\
\hline C. Sample & $8.60 \pm 0.00^{\mathrm{b}}$ & $0.30 \pm 0.00^{c}$ & $10.40 \pm 0.00^{c}$ & $24.60 \pm 0.00^{\mathrm{b}}$ & $\mathrm{BDL} \pm 0.00^{\mathrm{a}}$ & $\mathrm{BDL} \pm 0.00^{\mathrm{a}}$ \\
\hline \multicolumn{7}{|c|}{ Colocacia antiquorm } \\
\hline Q. Sample & $177.41 \pm 0.11^{\mathrm{c}}$ & $\mathrm{BDL} \pm 0.00^{\mathrm{a}}$ & $7.05 \pm 0.05^{\mathrm{b}}$ & $1274.85 \pm 0.15^{\mathrm{c}}$ & $\mathrm{BDL} \pm 0.00^{\mathrm{a}}$ & $\mathrm{BDL} \pm 0.00^{\mathrm{a}}$ \\
\hline C. Sample & $35.40 \pm 0.00^{\mathrm{b}}$ & $\mathrm{BDL} \pm 0.00^{\mathrm{a}}$ & $28.10 \pm 0.00^{\mathrm{c}}$ & $35.90 \pm 0.00^{\mathrm{b}}$ & $\mathrm{BDL} \pm 0.00^{\mathrm{a}}$ & $\mathrm{BDL} \pm 0.00^{\mathrm{a}}$ \\
\hline \multicolumn{7}{|c|}{ Telferia Occidentalis } \\
\hline Q. Sample & $10.16 \pm 0.06^{\mathrm{b}}$ & $\mathrm{BDL} \pm 0.00^{\mathrm{a}}$ & $12.51 \pm 0.01^{\mathrm{c}}$ & $174.13 \pm 0.03^{b}$ & $0.41 \pm 0.01^{\mathrm{b}}$ & $\mathrm{BDL} \pm 0.00^{\mathrm{a}}$ \\
\hline C. Sample & $39.80 \pm 0.00^{\mathrm{c}}$ & $\mathrm{BDL} \pm 0.00^{\mathrm{a}}$ & $11.10 \pm 0.00^{\mathrm{b}}$ & $213.00 \pm 0.00^{c}$ & $4.40 \pm 0.00^{c}$ & $\mathrm{BDL} \pm 0.00^{\mathrm{a}}$ \\
\hline \multicolumn{7}{|c|}{ Vernonia amygdalina } \\
\hline Q. Sample & $96.80 \pm 0.20^{\mathrm{c}}$ & $\mathrm{BDL} \pm 0.00^{\mathrm{a}}$ & $18.11 \pm 0.11^{\mathrm{c}}$ & $148.18 \pm 0.18^{c}$ & $\mathrm{BDL} \pm 0.00^{\mathrm{a}}$ & $\mathrm{BDL} \pm 0.00^{\mathrm{a}}$ \\
\hline C. Sample & $11.70 \pm 0.00^{\mathrm{b}}$ & $\mathrm{BDL} \pm 0.00^{\mathrm{a}}$ & $7.90 \pm 0.00^{\mathrm{b}}$ & $5.40 \pm 0.00^{\mathrm{b}}$ & $\mathrm{BDL} \pm 0.00^{\mathrm{a}}$ & $\mathrm{BDL} \pm 0.00^{\mathrm{a}}$ \\
\hline \multicolumn{7}{|c|}{ Talinum triangulare } \\
\hline Q. Sample & $40.84 \pm 0.14^{b}$ & $\mathrm{BDL} \pm 0.00^{\mathrm{a}}$ & $27.71 \pm 0.01^{\mathrm{c}}$ & $630.25 \pm 0.25^{b}$ & $\mathrm{BDL} \pm 0.00^{\mathrm{a}}$ & $15.65 \pm 0.15^{\mathrm{c}}$ \\
\hline C. Sample & $86.20 \pm 0.00^{c}$ & $\mathrm{BDL} \pm 0.00^{\mathrm{a}}$ & $5.40 \pm 0.00^{\mathrm{b}}$ & $906.40 \pm 0.00^{c}$ & $\mathrm{BDL} \pm 0.00^{\mathrm{a}}$ & $\mathrm{BDL} \pm 0.00^{\mathrm{b}}$ \\
\hline \multicolumn{7}{|l|}{ Citrus sinensis } \\
\hline Q. Sample & $10.45 \pm 0.07^{\mathrm{b}}$ & $\mathrm{BDL} \pm 0.00^{\mathrm{a}}$ & $13.75 \pm 0.25^{\mathrm{c}}$ & $211.35 \pm 0.15^{\mathrm{c}}$ & $212.7 \pm 0.06^{\mathrm{b}}$ & $25.95 \pm 0.05^{\mathrm{c}}$ \\
\hline C. Sample & $11.00 \pm 0.00^{c}$ & $\mathrm{BDL} \pm 0.00^{\mathrm{a}}$ & $7.40 \pm 0.00^{\mathrm{b}}$ & $63.10 \pm 0.00^{\mathrm{b}}$ & $0.30 \pm 0.00^{\mathrm{a}}$ & $\mathrm{BDL} \pm 0.00^{\mathrm{b}}$ \\
\hline \multicolumn{7}{|c|}{ Chrysophyllum albidun } \\
\hline Q. Sample & $11.40 \pm 0.00^{\mathrm{b}}$ & $\mathrm{BDL} \pm 0.00^{\mathrm{a}}$ & $21.40 \pm 0.10^{c}$ & $283.40 \pm 0.10^{\mathrm{b}}$ & $\mathrm{BDL} \pm 0.00^{\mathrm{a}}$ & $37.50 \pm 0.00^{\mathrm{c}}$ \\
\hline C. Sample & $204.80 \pm 0.00^{c}$ & $\mathrm{BDL} \pm 0.00^{\mathrm{a}}$ & $10.60 \pm 0.00^{\mathrm{b}}$ & $827.00 \pm 0.00^{c}$ & $\mathrm{BDL} \pm 0.00^{\mathrm{a}}$ & $\mathrm{BDL} \pm 0.00^{\mathrm{b}}$ \\
\hline \multicolumn{7}{|l|}{ Carica papaya } \\
\hline Q. Sample & $35.21 \pm 0.01^{\mathrm{c}}$ & $\mathrm{BDL} \pm 0.00^{\mathrm{a}}$ & $15.81 \pm 0.11^{\mathrm{b}}$ & $1455.25 \pm 4.25^{\mathrm{c}}$ & $2.90 \pm 0.10^{\mathrm{b}}$ & $80.21 \pm 0.01^{\mathrm{c}}$ \\
\hline C. Sample & $0.20 \pm 0.00^{\mathrm{b}}$ & $\mathrm{BDL} \pm 0.00^{\mathrm{a}}$ & $3.00 \pm 0.00^{\mathrm{a}}$ & $7.70 \pm 0.00^{\mathrm{b}}$ & $\mathrm{BDL} \pm 0.00^{\mathrm{a}}$ & $\mathrm{BDL} \pm 0.00^{\mathrm{b}}$ \\
\hline \multicolumn{7}{|l|}{ Cocos nucifera } \\
\hline Q. Sample & $53.40 \pm 0.10^{\mathrm{b}}$ & $\mathrm{BDL} \pm 0.00^{\mathrm{a}}$ & $7.40 \pm 0.10^{\mathrm{b}}$ & $314.95 \pm 0.05^{\mathrm{b}}$ & $\mathrm{BDL} \pm 0.00^{\mathrm{a}}$ & $\mathrm{BDL} \pm 0.00^{\mathrm{a}}$ \\
\hline C. Sample & $184.50 \pm 0.00^{\mathrm{c}}$ & $\mathrm{BDL} \pm 0.00^{\mathrm{a}}$ & $7.90 \pm 0.00^{\mathrm{b}}$ & $742.90 \pm 0.00^{c}$ & $\mathrm{BDL} \pm 0.00^{\mathrm{a}}$ & $\mathrm{BDL} \pm 0.00^{\mathrm{a}}$ \\
\hline \multicolumn{7}{|l|}{ Cola acuminata } \\
\hline Q. Sample & $147.85 \pm 0.05^{\mathrm{c}}$ & $\mathrm{BDL} \pm 0.00^{\mathrm{a}}$ & $7.35 \pm 0.15^{\mathrm{a}}$ & $833.22 \pm 0.02^{\mathrm{c}}$ & $\mathrm{BDL} \pm 0.00^{\mathrm{a}}$ & $\mathrm{BDL} \pm 0.00^{\mathrm{a}}$ \\
\hline C. Sample & $9.20 \pm 0.00^{\mathrm{b}}$ & $\mathrm{BDL} \pm 0.00^{\mathrm{a}}$ & $9.20 \pm 0.00^{\mathrm{b}}$ & $55.50 \pm 0.00^{\mathrm{b}}$ & $\mathrm{BDL} \pm 0.00^{\mathrm{a}}$ & $\mathrm{BDL} \pm 0.00^{\mathrm{a}}$ \\
\hline \multicolumn{7}{|c|}{ Elaies guineensis } \\
\hline Q. Sample & $24.90 \pm 0.10^{\mathrm{c}}$ & $\mathrm{BDL} \pm 0.00^{\mathrm{a}}$ & $6.35 \pm 0.15^{\mathrm{b}}$ & $31.95 \pm 0.05^{\mathrm{b}}$ & $\mathrm{BDL} \pm 0.00^{\mathrm{a}}$ & $\mathrm{BDL} \pm 0.00^{\mathrm{a}}$ \\
\hline C. Sample & $8.10 \pm 0.00^{\mathrm{b}}$ & $\mathrm{BDL} \pm 0.00^{\mathrm{a}}$ & $3.20 \pm 0.00^{\mathrm{a}}$ & $92.60 \pm 0.00^{c}$ & $2.40 \pm 0.00^{\mathrm{b}}$ & $\mathrm{BDL} \pm 0.00^{\mathrm{a}}$ \\
\hline \multicolumn{7}{|l|}{ Total } \\
\hline Ishiagu & 760.81 & 0.236 & 144.745 & $8,275.72$ & 216.878 & 159.313 \\
\hline Umudike & 845.4 & 0.311 & 110.3 & 4470.5 & 7.413 & $\mathrm{BDL} \pm 0.00$ \\
\hline
\end{tabular}

Values are expressed as mean \pm SEM of triplicate $(n=3)$.

$\mathrm{a}=$ there is no significant difference in mean metal concentration between control and test sample at $(p>0.05)$.

$\mathrm{b}=$ there is significant increase in mean metal concentration between control and test sample at $(p<0.05)$.

$\mathrm{c}=$ there is significant decrease in mean metal concentration between control and test sample at $(p<0.05)$.

Q. Sample $=$ Sample from Quarry site

C. Sample $=$ Sample from Control site

Table 4. Mean concentration of heavy metals ( $\mathrm{mg} / \mathrm{kg}$ dry weight) in rhizosphere soils from Quarry site (Ishiagu) and residential area (Umudike)

\begin{tabular}{|c|c|c|c|c|c|c|}
\hline $\begin{array}{l}\text { Heavy } \\
\text { metals }\end{array}$ & Mn & Cd & $\mathbf{Z n}$ & $\mathrm{Fe}$ & $\mathbf{P b}$ & $\mathbf{C u}$ \\
\hline \multicolumn{7}{|c|}{ Manihot esculenta } \\
\hline Q. Soil & $65.55 \pm 0.05^{\mathrm{c}}$ & $\mathrm{BDL} \pm 0.00^{\mathrm{a}}$ & $2.70 \pm 0.10^{\mathrm{b}}$ & $707.25 \pm 0.25^{\mathrm{b}}$ & $\mathrm{BDL} \pm 0.00^{\mathrm{b}}$ & $\mathrm{BDL} \pm 0.00^{\mathrm{a}}$ \\
\hline C. Soil & $26.51 \pm 0.01^{\mathrm{b}}$ & $\mathrm{BDL} \pm 0.00^{\mathrm{a}}$ & $6.61 \pm 0.01^{\mathrm{c}}$ & $911.11 \pm 0.01^{\mathrm{c}}$ & $3.72 \pm 0.02^{\mathrm{c}}$ & $\mathrm{BDL} \pm 0.00^{\mathrm{a}}$ \\
\hline \multicolumn{7}{|c|}{ Dioscorea rodundata } \\
\hline Q. Soil & $119.55 \pm 0.05^{\mathrm{b}}$ & $\mathrm{BDL} \pm 0.00^{\mathrm{a}}$ & $19.85 \pm 0.15^{\mathrm{a}}$ & $6347.90 \pm 0.0^{c}$ & $9.80 \pm 0.20^{\mathrm{c}}$ & $4.70 \pm 0.10^{c}$ \\
\hline C. Soil & $209.81 \pm 0.01^{\mathrm{c}}$ & $\mathrm{BDL} \pm 0.00 \mathrm{a}$ & $19.88 \pm 0.03^{\mathrm{a}}$ & $2647.41 \pm 0.1^{\mathrm{b}}$ & $\mathrm{BDL} \pm 0.00^{\mathrm{b}}$ & $\mathrm{BDL} \pm 0.00^{\mathrm{b}}$ \\
\hline \multicolumn{7}{|c|}{ Colocacia antiquorm } \\
\hline Q. Soil & $183.30 \pm 0.20^{b}$ & $\mathrm{BDL} \pm 0.00^{\mathrm{a}}$ & $53.95 \pm 0.05^{\mathrm{c}}$ & $7148.45 \pm 0.05^{\mathrm{b}}$ & $\mathrm{BDL} \pm 0.00^{\mathrm{a}}$ & $1.35 \pm 0.15^{\mathrm{b}}$ \\
\hline C. Soil & $275.60 \pm 0.00^{c}$ & $\mathrm{BDL} \pm 0.00^{\mathrm{a}}$ & $29.20 \pm 0.00^{\mathrm{b}}$ & $7301.20 \pm 0.00^{c}$ & $\mathrm{BDL} \pm 0.00^{\mathrm{a}}$ & $49.90 \pm 0.00^{c}$ \\
\hline
\end{tabular}




\begin{tabular}{|c|c|c|c|c|c|c|}
\hline $\begin{array}{l}\text { Heavy } \\
\text { metals }\end{array}$ & Mn & $\mathbf{C d}$ & $\mathbf{Z n}$ & $\mathbf{F e}$ & $\mathbf{P b}$ & $\mathbf{C u}$ \\
\hline \multicolumn{7}{|c|}{ Telferia Occidentalis } \\
\hline Q. Soil & $91.75 \pm 0.05^{\mathrm{c}}$ & $\mathrm{BDL} \pm 0.00^{\mathrm{a}}$ & $18.75 \pm 0.5^{\mathrm{c}}$ & $6590.50 \pm 0.10^{c}$ & $\mathrm{BDL} \pm 0.00^{\mathrm{b}}$ & $\mathrm{BDL} \pm 0.00^{\mathrm{a}}$ \\
\hline C. Soil & $6.25 \pm 0.05^{\mathrm{b}}$ & $\mathrm{BDL} \pm 0.00^{\mathrm{a}}$ & $9.11 \pm 0.01^{\mathrm{b}}$ & $899.31 \pm 0.01^{\mathrm{b}}$ & $3.00 \pm 0.00^{\mathrm{c}}$ & $\mathrm{BDL} \pm 0.00^{\mathrm{a}}$ \\
\hline \multicolumn{7}{|c|}{ Vernonia amygdalina } \\
\hline Q. Soil & $248.50 \pm 0.10^{\mathrm{c}}$ & $\mathrm{BDL} \pm 0.00^{\mathrm{a}}$ & $18.10 \pm 0.10^{\mathrm{c}}$ & $148.00 \pm 0.00^{\mathrm{b}}$ & $\mathrm{BDL} \pm 0.00^{\mathrm{a}}$ & $\mathrm{BDL} \pm 0.00^{\mathrm{a}}$ \\
\hline C. Soil & $30.81 \pm 0.01^{\mathrm{b}}$ & $\mathrm{BDL} \pm 0.00^{\mathrm{a}}$ & $25.91 \pm 0.01^{\mathrm{b}}$ & $1619.61 \pm 0.01^{\mathrm{c}}$ & $\mathrm{BDL} \pm 0.00^{\mathrm{a}}$ & $\mathrm{BDL} \pm 0.00^{\mathrm{a}}$ \\
\hline \multicolumn{7}{|c|}{ Talinum triangulare } \\
\hline Q. Soil & $179.50 \pm 0.10^{c}$ & $\mathrm{BDL} \pm 0.00^{\mathrm{a}}$ & $27.85 \pm 0.15^{\mathrm{c}}$ & $630.10 \pm 0.10^{\mathrm{b}}$ & $\mathrm{BDL} \pm 0.00^{\mathrm{a}}$ & $15.65 \pm 0.15^{\mathrm{c}}$ \\
\hline C. Soil & $8.50 \pm 0.00^{\mathrm{b}}$ & $\mathrm{BDL} \pm 0.00^{\mathrm{a}}$ & $5.40 \pm 0.00^{\mathrm{b}}$ & $906.40 \pm 0.00^{c}$ & $\mathrm{BDL} \pm 0.00^{\mathrm{a}}$ & $\mathrm{BDL} \pm 0.00^{\mathrm{b}}$ \\
\hline
\end{tabular}

Table 5. Bio-concentration factor of crop plants from Quarry site (Ishiagu) and agricultural zone (Umudike)

\begin{tabular}{|c|c|c|c|c|c|c|c|}
\hline Heavy metals & Mn & $\mathbf{C d}$ & $\mathbf{Z n}$ & $\mathbf{F e}$ & $\mathbf{P b}$ & $\mathbf{C u}$ & Total \\
\hline \multicolumn{8}{|c|}{ Manihot esculenta } \\
\hline Q. Sample & $1.80 \mathrm{E}+00$ & $2.25 \mathrm{E}+02$ & $1.52 \mathrm{E}+00$ & $3.75 \mathrm{E}+00$ & $9.10 \mathrm{E}+02$ & $1.00 \mathrm{E}+00$ & $1.14 \mathrm{E}+03$ \\
\hline Sample Control & $9.28 \mathrm{E}+00$ & $1.00 \mathrm{E}+00$ & $9.23 \mathrm{E}-01$ & $1.64 \mathrm{E}+00$ & 2.69E-04 & $1.00 \mathrm{E}+00$ & $1.38 \mathrm{E}+01$ \\
\hline \multicolumn{8}{|c|}{ Dioscorea rodundata } \\
\hline Q. Sample & $2.85 \mathrm{E}-01$ & $1.00 \mathrm{E}+00$ & $1.62 \mathrm{E}-01$ & 4.16E-02 & $1.02 \mathrm{E}-04$ & $2.13 \mathrm{E}-04$ & $1.49 \mathrm{E}+00$ \\
\hline Sample Control & 4.10E-02 & $3.00 \mathrm{E}+02$ & $5.23 \mathrm{E}-01$ & $9.29 \mathrm{E}-03$ & $1.00 \mathrm{E}+00$ & $1.00 \mathrm{E}+00$ & $3.03 \mathrm{E}+02$ \\
\hline \multicolumn{8}{|c|}{ Colocacia antiquorm } \\
\hline Q. Sample & $9.68 \mathrm{E}-01$ & $1.00 \mathrm{E}+00$ & $1.31 \mathrm{E}-01$ & $1.78 \mathrm{E}-01$ & $1.00 \mathrm{E}+00$ & $7.41 \mathrm{E}-04$ & $3.28 \mathrm{E}+00$ \\
\hline Sample Control & $1.28 \mathrm{E}-01$ & $1.00 \mathrm{E}+00$ & $9.62 \mathrm{E}-01$ & $4.92 \mathrm{E}-03$ & $1.00 \mathrm{E}+00$ & $2.00 \mathrm{E}-05$ & $3.09 \mathrm{E}+00$ \\
\hline \multicolumn{8}{|c|}{ Telferia Occidentalis } \\
\hline Q. Sample & $1.11 \mathrm{E}-01$ & $1.00 \mathrm{E}+00$ & $6.67 \mathrm{E}-01$ & $2.64 \mathrm{E}-02$ & $4.05 \mathrm{E}+02$ & $1.00 \mathrm{E}+00$ & $4.08 \mathrm{E}+02$ \\
\hline Sample Control & $6.37 \mathrm{E}+00$ & $1.00 \mathrm{E}+00$ & $1.22 \mathrm{E}+00$ & $2.37 \mathrm{E}-01$ & $1.47 \mathrm{E}+00$ & $1.00 \mathrm{E}+00$ & $1.13 \mathrm{E}+01$ \\
\hline \multicolumn{8}{|c|}{ Vernonia amygdalina } \\
\hline Q. Sample & 3.90E-01 & $1.00 \mathrm{E}+00$ & $1.00 \mathrm{E}+00$ & $1.00 \mathrm{E}+00$ & $1.00 \mathrm{E}+00$ & $1.00 \mathrm{E}+00$ & $5.39 \mathrm{E}+00$ \\
\hline Sample Control & $3.80 \mathrm{E}-01$ & $1.00 \mathrm{E}+00$ & $3.05 \mathrm{E}-01$ & $3.33 \mathrm{E}-03$ & $1.00 \mathrm{E}+00$ & $1.00 \mathrm{E}+00$ & $3.69 \mathrm{E}+00$ \\
\hline \multicolumn{8}{|c|}{ Talinum triangulare } \\
\hline Q. Sample & $2.28 \mathrm{E}-01$ & $1.00 \mathrm{E}+00$ & $9.95 \mathrm{E}-01$ & $1.00 \mathrm{E}+00$ & $1.00 \mathrm{E}+00$ & $1.00 \mathrm{E}+00$ & $5.22 \mathrm{E}+00$ \\
\hline Sample Control & $1.01 \mathrm{E}+01$ & $1.00 \mathrm{E}+00$ & $1.00 \mathrm{E}+00$ & $1.00 \mathrm{E}+00$ & $1.00 \mathrm{E}+00$ & $1.00 \mathrm{E}+00$ & $1.51 \mathrm{E}+01$ \\
\hline \multicolumn{8}{|l|}{ Total } \\
\hline Ishiagu & $3.78 \mathrm{E}+00$ & $2.30 \mathrm{E}+02$ & $4.48 \mathrm{E}+00$ & $6.00 \mathrm{E}+00$ & $1.32 \mathrm{E}+03$ & $4.00 \mathrm{E}+00$ & $1.57 \mathrm{E}+03$ \\
\hline Umudike & $2.63 \mathrm{E}+01$ & $3.05 \mathrm{E}+02$ & $4.93 \mathrm{E}+00$ & $2.89 \mathrm{E}+00$ & $5.47 \mathrm{E}+00$ & $5.00 \mathrm{E}+00$ & $3.50 \mathrm{E}+02$ \\
\hline
\end{tabular}

Table 6. Daily intake $\left(\mathrm{mg} \mathrm{kg}^{-1} \mathrm{day}^{-1}\right)$ of heavy metals by adults who reside around quarry sites (Ishiagu) and agricultural area (Umudike)

\begin{tabular}{|c|c|c|c|c|c|c|c|}
\hline $\begin{array}{l}\text { Heavy } \\
\text { metals }\end{array}$ & Mn & Cd & $\mathbf{Z n}$ & $\mathbf{F e}$ & $\mathbf{P b}$ & $\mathbf{C u}$ & Total \\
\hline \multicolumn{8}{|c|}{ Manihot esculenta } \\
\hline Q. Sample & 7.01E-02 & $1.33 \mathrm{E}-04$ & $2.43 \mathrm{E}-03$ & $1.57 \mathrm{E}+00$ & 5.39E-04 & $5.92 \mathrm{E}-07$ & $1.64 \mathrm{E}+00$ \\
\hline Control & $1.46 \mathrm{E}-01$ & $5.92 \mathrm{E}-07$ & $3.61 \mathrm{E}-03$ & $8.86 \mathrm{E}-01$ & $5.92 \mathrm{E}-07$ & $5.92 \mathrm{E}-07$ & $1.04 \mathrm{E}+00$ \\
\hline \multicolumn{8}{|c|}{ Dioscorea rodundata } \\
\hline Q. Sample & $2.02 \mathrm{E}-02$ & $5.92 \mathrm{E}-07$ & $1.91 \mathrm{E}-03$ & $1.56 \mathrm{E}-01$ & 5.92E-07 & 5.92E-07 & $1.78 \mathrm{E}-01$ \\
\hline Control & 5.09E-03 & $1.78 \mathrm{E}-04$ & $6.16 \mathrm{E}-03$ & $1.46 \mathrm{E}-02$ & 5.92E-07 & 5.92E-07 & $2.60 \mathrm{E}-02$ \\
\hline \multicolumn{8}{|c|}{ Colocacia antiquorm } \\
\hline Q. Sample & $1.05 \mathrm{E}-01$ & $5.92 \mathrm{E}-07$ & 4.17E-03 & $7.55 \mathrm{E}-01$ & $5.92 \mathrm{E}-07$ & $5.88 \mathrm{E}-07$ & $8.64 \mathrm{E}-01$ \\
\hline Control & $2.10 \mathrm{E}-02$ & $5.92 \mathrm{E}-07$ & $1.66 \mathrm{E}-02$ & $2.13 \mathrm{E}-02$ & 5.92E-07 & 5.88E-07 & $5.89 \mathrm{E}-02$ \\
\hline \multicolumn{8}{|c|}{ Telferia Occidentalis } \\
\hline Q. Sample & 4.96E-03 & 4.89E-07 & $6.11 \mathrm{E}-03$ & $8.51 \mathrm{E}-02$ & $1.98 \mathrm{E}-04$ & 4.89E-07 & $9.64 \mathrm{E}-02$ \\
\hline Control & $1.95 \mathrm{E}-02$ & 4.89E-07 & $5.43 \mathrm{E}-03$ & $1.04 \mathrm{E}-01$ & $2.15 \mathrm{E}-03$ & 4.89E-07 & $1.31 \mathrm{E}-01$ \\
\hline
\end{tabular}




\begin{tabular}{|c|c|c|c|c|c|c|c|}
\hline \multicolumn{8}{|c|}{ Vernonia amygdalina } \\
\hline Q. Sample & $4.73 \mathrm{E}-02$ & $4.89 \mathrm{E}-07$ & $8.85 \mathrm{E}-03$ & 7.24E-02 & $4.89 \mathrm{E}-07$ & 4.89E-07 & $1.29 \mathrm{E}-01$ \\
\hline Control & $5.72 \mathrm{E}-03$ & $4.89 \mathrm{E}-07$ & $3.86 \mathrm{E}-03$ & $2.64 \mathrm{E}-03$ & 4.89E-07 & 4.89E-07 & $1.22 \mathrm{E}-02$ \\
\hline \multicolumn{8}{|c|}{ Talinum triangulare } \\
\hline Q. Sample & $2.00 \mathrm{E}-02$ & $4.89 \mathrm{E}-07$ & $1.35 \mathrm{E}-02$ & $3.08 \mathrm{E}-01$ & 4.89E-07 & 7.65E-03 & $3.49 \mathrm{E}-01$ \\
\hline Control & $4.21 \mathrm{E}-02$ & 4.89E-07 & $2.64 \mathrm{E}-03$ & 4.43E-01 & $4.89 \mathrm{E}-07$ & 4.89E-07 & $4.88 \mathrm{E}-01$ \\
\hline \multicolumn{8}{|c|}{ Citrus sinensis } \\
\hline Q. Sample & $5.11 \mathrm{E}-03$ & $4.89 \mathrm{E}-07$ & $6.72 \mathrm{E}-03$ & $1.03 \mathrm{E}-01$ & $1.04 \mathrm{E}-01$ & $1.27 \mathrm{E}-02$ & 2.32E-01 \\
\hline Control & $5.38 \mathrm{E}-03$ & $4.89 \mathrm{E}-07$ & $3.62 \mathrm{E}-03$ & $3.08 \mathrm{E}-02$ & $1.47 \mathrm{E}-04$ & 4.89E-07 & 3.99E-02 \\
\hline \multicolumn{8}{|c|}{ Chrysophyllum albidun } \\
\hline Q. Sample & $5.57 \mathrm{E}-03$ & $4.8 \mathrm{~s}$ & 02 & 1.39 & 97 & 22 & -01 \\
\hline Control & $1.00 \mathrm{E}-01$ & $4.8 \mathrm{~s}$ & 03 & 4.0 & 4.8 & 4.8 & $5.09 \mathrm{E}-01$ \\
\hline \multicolumn{8}{|c|}{ Carica papaya } \\
\hline Q. Sample & 1.72 & 4.8 & 7.7 & 7.1 & & 02 & 7.77E-01 \\
\hline Control & $9.78 \mathrm{E}-05$ & $4.89 \mathrm{E}-07$ & $1.47 \mathrm{E}-03$ & $3.76 \mathrm{E}-03$ & 4.89E-07 & 4.89E-07 & $5.33 \mathrm{E}-03$ \\
\hline \multicolumn{8}{|c|}{ Cocos nucifera } \\
\hline Q. Sample & $2.61 \mathrm{E}-02$ & 4.89 & $3.62 \mathrm{E}-03$ & 1.5 & 4.8 & 4.89E-07 & $1.84 \mathrm{E}-01$ \\
\hline Control & $9.02 \mathrm{E}-02$ & $4.89 \mathrm{E}-07$ & 3.86 & 3.6 & 4.8 & 4.8 & 4.57E-01 \\
\hline \multicolumn{8}{|c|}{ Cola acuminata } \\
\hline Q. Sample & $7.23 \mathrm{E}-02$ & $4.89 \mathrm{E}-07$ & $3.59 \mathrm{E}-03$ & 4.07E-01 & 4.89E-07 & 4.89E-07 & 4.83E-01 \\
\hline Control & $4.50 \mathrm{E}-03$ & $4.89 \mathrm{E}-07$ & $4.50 \mathrm{E}-03$ & $2.71 \mathrm{E}-02$ & 4.89E-07 & 4.89E-07 & $3.61 \mathrm{E}-02$ \\
\hline \multicolumn{8}{|c|}{ Elaies guineensis } \\
\hline Q. Sample & $1.22 \mathrm{E}-02$ & $4.89 \mathrm{E}$ & 3.10 & 1.56 & $4.89 \mathrm{I}$ & 4.89E-07 & $3.09 \mathrm{E}-02$ \\
\hline Control & $3.96 \mathrm{E}-03$ & 4.89E-07 & $1.56 \mathrm{E}-03$ & $4.53 \mathrm{E}-02$ & $1.17 \mathrm{E}-03$ & 4.89E-07 & $5.20 \mathrm{E}-02$ \\
\hline \multicolumn{8}{|l|}{ Average } \\
\hline Quarry site & $3.38 \mathrm{E}-02$ & 1.15 & $6.02 \mathrm{E}-03$ & $3.73 \mathrm{E}-01$ & $8.85 \mathrm{E}-03$ & $6.49 \mathrm{E}-03$ & $4.28 \mathrm{E}-01$ \\
\hline Control site & $3.70 \mathrm{E}-02$ & $1.53 \mathrm{E}-05$ & $4.87 \mathrm{E}-03$ & $1.95 \mathrm{E}-01$ & $2.89 \mathrm{E}-04$ & 5.14E-07 & $2.38 \mathrm{E}-01$ \\
\hline
\end{tabular}

Table 7. Daily intake $\left(\mathrm{mg} \mathrm{kg}^{-1} \mathrm{day}^{-1}\right)$ of heavy metals by children who reside around quarry sites (Ishiagu) and agricultural area (Umudike)

\begin{tabular}{|c|c|c|c|c|c|c|c|}
\hline Heavy metals & Mn & Cd & $\mathbf{Z n}$ & $\mathbf{F e}$ & $\mathbf{P b}$ & $\mathbf{C u}$ & Total \\
\hline \multicolumn{8}{|c|}{ Manihot esculenta } \\
\hline Q. Sample & 7.01E-02 & $1.33 \mathrm{E}-04$ & $2.43 \mathrm{E}-03$ & $1.57 \mathrm{E}+00$ & 5.39E-04 & 5.92E-07 & $1.64 \mathrm{E}+00$ \\
\hline Control & $1.46 \mathrm{E}-01$ & 5.92E-07 & $3.61 \mathrm{E}-03$ & $8.86 \mathrm{E}-01$ & 5.92E-07 & $5.92 \mathrm{E}-07$ & $1.04 \mathrm{E}+00$ \\
\hline \multicolumn{8}{|c|}{ Dioscorea rodundata } \\
\hline Q. Sample & $2.02 \mathrm{E}-02$ & 5.92E-07 & $1.91 \mathrm{E}-03$ & $1.56 \mathrm{E}-01$ & 5.92E-07 & $5.92 \mathrm{E}-07$ & $1.78 \mathrm{E}-01$ \\
\hline Control & $5.09 \mathrm{E}-03$ & $1.78 \mathrm{E}-04$ & $6.16 \mathrm{E}-03$ & $1.46 \mathrm{E}-02$ & 5.92E-07 & $5.92 \mathrm{E}-07$ & $2.60 \mathrm{E}-02$ \\
\hline \multicolumn{8}{|c|}{ Colocacia antiquorm } \\
\hline Q. Sample & $1.05 \mathrm{E}-01$ & 5.92E-07 & $4.17 \mathrm{E}-03$ & $7.55 \mathrm{E}-01$ & 5.92E-07 & $5.88 \mathrm{E}-07$ & 8.64E-01 \\
\hline Control & $2.10 \mathrm{E}-02$ & $5.92 \mathrm{E}-07$ & $1.66 \mathrm{E}-02$ & $2.13 \mathrm{E}-02$ & 5.92E-07 & $5.88 \mathrm{E}-07$ & $5.89 \mathrm{E}-02$ \\
\hline \multicolumn{8}{|c|}{ Telferia Occidentalis } \\
\hline Q. Sample & $4.96 \mathrm{E}-03$ & 4.89E-07 & $6.11 \mathrm{E}-03$ & $8.51 \mathrm{E}-02$ & $1.98 \mathrm{E}-04$ & $4.89 \mathrm{E}-07$ & $9.64 \mathrm{E}-02$ \\
\hline Control & $1.95 \mathrm{E}-02$ & $4.89 \mathrm{E}-07$ & $5.43 \mathrm{E}-03$ & $1.04 \mathrm{E}-01$ & $2.15 \mathrm{E}-03$ & $4.89 \mathrm{E}-07$ & $1.31 \mathrm{E}-01$ \\
\hline \multicolumn{8}{|c|}{ Vernonia amygdalina } \\
\hline Q. Sample & 4.73E-02 & $4.89 \mathrm{E}-07$ & $8.85 \mathrm{E}-03$ & 7.24E-02 & $4.89 \mathrm{E}-07$ & $4.89 \mathrm{E}-07$ & $1.29 \mathrm{E}-01$ \\
\hline Control & $5.72 \mathrm{E}-03$ & $4.89 \mathrm{E}-07$ & $3.86 \mathrm{E}-03$ & $2.64 \mathrm{E}-03$ & $4.89 \mathrm{E}-07$ & $4.89 \mathrm{E}-07$ & $1.22 \mathrm{E}-02$ \\
\hline \multicolumn{8}{|c|}{ Talinum triangulare } \\
\hline Q. Sample & $2.00 \mathrm{E}-02$ & $4.89 \mathrm{E}-07$ & $1.35 \mathrm{E}-02$ & $3.08 \mathrm{E}-01$ & $4.89 \mathrm{E}-07$ & $7.65 \mathrm{E}-03$ & $3.49 \mathrm{E}-01$ \\
\hline Control & $4.21 \mathrm{E}-02$ & $4.89 \mathrm{E}-07$ & $2.64 \mathrm{E}-03$ & $4.43 \mathrm{E}-01$ & $4.89 \mathrm{E}-07$ & $4.89 \mathrm{E}-07$ & $4.88 \mathrm{E}-01$ \\
\hline \multicolumn{8}{|c|}{ Citrus sinensis } \\
\hline Q. Sample & $5.11 \mathrm{E}-03$ & 4.89E-07 & $6.72 \mathrm{E}-03$ & $1.03 \mathrm{E}-01$ & $1.04 \mathrm{E}-01$ & $1.27 \mathrm{E}-02$ & $2.32 \mathrm{E}-01$ \\
\hline Control & $5.38 \mathrm{E}-03$ & $4.89 \mathrm{E}-07$ & $3.62 \mathrm{E}-03$ & $3.08 \mathrm{E}-02$ & $1.47 \mathrm{E}-04$ & $4.89 \mathrm{E}-07$ & $3.99 \mathrm{E}-02$ \\
\hline \multicolumn{8}{|c|}{ Chrysophyllum albidun } \\
\hline Q. Sample & $5.57 \mathrm{E}-03$ & $4.89 \mathrm{E}-07$ & $1.05 \mathrm{E}-02$ & $1.39 \mathrm{E}-01$ & $4.89 \mathrm{E}-07$ & $1.83 \mathrm{E}-02$ & $1.73 \mathrm{E}-01$ \\
\hline Control & $1.00 \mathrm{E}-01$ & 4.89E-07 & $5.18 \mathrm{E}-03$ & $4.04 \mathrm{E}-01$ & 4.89E-07 & $4.89 \mathrm{E}-07$ & $5.09 \mathrm{E}-01$ \\
\hline
\end{tabular}




\begin{tabular}{|c|c|c|c|c|c|c|c|}
\hline \multicolumn{8}{|c|}{ Carica papaya } \\
\hline Q. Sample & 1.72E-02 & 4.89E-07 & 7.72E-03 & 7.11E-01 & $1.42 \mathrm{E}-03$ & $3.92 \mathrm{E}-02$ & 7.77E-01 \\
\hline Control & $9.78 \mathrm{E}-05$ & 4.89E-07 & $1.47 \mathrm{E}-03$ & $3.76 \mathrm{E}-03$ & 4.89E-07 & $4.89 \mathrm{E}-07$ & $5.33 \mathrm{E}-03$ \\
\hline \multicolumn{8}{|c|}{ Cocos nucifera } \\
\hline Q. Sample & 2.61E-02 & 4.89E-07 & $3.62 \mathrm{E}-03$ & $1.54 \mathrm{E}-01$ & 4.89E-07 & 4.89E-07 & $1.84 \mathrm{E}-01$ \\
\hline Control & $9.02 \mathrm{E}-02$ & 4.89E-07 & $3.86 \mathrm{E}-03$ & $3.63 \mathrm{E}-01$ & 4.89E-07 & 4.89E-07 & $4.57 \mathrm{E}-01$ \\
\hline \multicolumn{8}{|c|}{ Cola acuminata } \\
\hline Q. Sample & 7.23E-02 & 4.89E-07 & $3.59 \mathrm{E}-03$ & 4.07E-01 & 4.89E-07 & 4.89E-07 & 4.83E-01 \\
\hline Control & $4.50 \mathrm{E}-03$ & 4.89E-07 & $4.50 \mathrm{E}-03$ & $2.71 \mathrm{E}-02$ & 4.89E-07 & $4.89 \mathrm{E}-07$ & 3.61E-02 \\
\hline \multicolumn{8}{|c|}{ Elaies guineensis } \\
\hline Q. Sample & $1.22 \mathrm{E}-02$ & 4.89E-07 & $3.10 \mathrm{E}-03$ & $1.56 \mathrm{E}-02$ & 4.89E-07 & $4.89 \mathrm{E}-07$ & $3.09 \mathrm{E}-02$ \\
\hline Control & $3.96 \mathrm{E}-03$ & 4.89E-07 & $1.56 \mathrm{E}-03$ & $4.53 \mathrm{E}-02$ & $1.17 \mathrm{E}-03$ & $4.89 \mathrm{E}-07$ & $5.20 \mathrm{E}-02$ \\
\hline \multicolumn{8}{|l|}{ Average } \\
\hline Quarry site & $3.38 \mathrm{E}-02$ & $1.15 \mathrm{E}-05$ & $6.02 \mathrm{E}-03$ & $3.73 \mathrm{E}-01$ & $8.85 \mathrm{E}-03$ & $6.49 \mathrm{E}-03$ & $4.28 \mathrm{E}-01$ \\
\hline Control site & $3.70 \mathrm{E}-02$ & $1.53 \mathrm{E}-05$ & $4.87 \mathrm{E}-03$ & $1.95 \mathrm{E}-01$ & 2.89E-04 & $5.14 \mathrm{E}-07$ & $2.38 \mathrm{E}-01$ \\
\hline
\end{tabular}

Table 8. Target hazard quotient and hazardous indexes of heavy metals in selected food crops for adults who live around quarry sites (Ishiagu) and agricultural area (Umudike)

\begin{tabular}{|c|c|c|c|c|c|c|c|}
\hline Heavy metals & Mn & Cd & $\mathbf{Z n}$ & $\mathbf{F e}$ & $\mathbf{P b}$ & $\mathbf{C u}$ & $\begin{array}{l}\text { Hazardous } \\
\text { index }\end{array}$ \\
\hline \multicolumn{8}{|c|}{ Manihot esculenta } \\
\hline Q. Sample & $5.00 \mathrm{E}+00$ & $1.33 \mathrm{E}-01$ & 8.09E-03 & $1.96 \mathrm{E}+00$ & $1.54 \mathrm{E}-01$ & $1.48 \mathrm{E}-05$ & $7.26 \mathrm{E}+00$ \\
\hline Sample Control & $1.04 \mathrm{E}+01$ & $5.92 \mathrm{E}-04$ & $1.20 \mathrm{E}-02$ & $1.11 \mathrm{E}+00$ & $1.69 \mathrm{E}-04$ & $1.48 \mathrm{E}-05$ & $1.15 \mathrm{E}+01$ \\
\hline \multicolumn{8}{|c|}{ Dioscorea rodundata } \\
\hline Q. Sample & $1.44 \mathrm{E}+00$ & $5.92 \mathrm{E}-04$ & $6.37 \mathrm{E}-03$ & $1.95 \mathrm{E}-01$ & $1.69 \mathrm{E}-04$ & $1.48 \mathrm{E}-05$ & $1.64 \mathrm{E}+00$ \\
\hline Sample Control & 3.64E-01 & $1.78 \mathrm{E}-01$ & $2.05 \mathrm{E}-02$ & $1.82 \mathrm{E}-02$ & $1.69 \mathrm{E}-04$ & $1.48 \mathrm{E}-05$ & $5.81 \mathrm{E}-01$ \\
\hline \multicolumn{8}{|c|}{ Colocacia antiquorm } \\
\hline Q. Sample & $7.50 \mathrm{E}+00$ & $5.92 \mathrm{E}-04$ & $1.39 \mathrm{E}-02$ & $9.44 \mathrm{E}-01$ & $1.69 \mathrm{E}-04$ & $1.47 \mathrm{E}-05$ & $8.46 \mathrm{E}+00$ \\
\hline Sample Control & $1.50 \mathrm{E}+00$ & 5.92E-04 & $5.55 \mathrm{E}-02$ & $2.66 \mathrm{E}-02$ & $1.69 \mathrm{E}-04$ & $1.47 \mathrm{E}-05$ & $1.58 \mathrm{E}+00$ \\
\hline \multicolumn{8}{|c|}{ Telferia Occidentalis } \\
\hline Q. Sample & $3.55 \mathrm{E}-01$ & 4.89E-04 & $2.04 \mathrm{E}-02$ & $1.06 \mathrm{E}-01$ & $5.66 \mathrm{E}-02$ & $1.22 \mathrm{E}-05$ & $5.39 \mathrm{E}-01$ \\
\hline Sample Control & $1.39 \mathrm{E}+00$ & 4.89E-04 & $1.81 \mathrm{E}-02$ & $1.30 \mathrm{E}-01$ & $6.14 \mathrm{E}-01$ & $1.22 \mathrm{E}-05$ & $2.15 \mathrm{E}+00$ \\
\hline \multicolumn{8}{|c|}{ Vernonia amygdalina } \\
\hline Q. Sample & $3.38 \mathrm{E}+00$ & 4.89E-04 & 2.95E-02 & $9.05 \mathrm{E}-02$ & $1.40 \mathrm{E}-04$ & $1.22 \mathrm{E}-05$ & $3.50 \mathrm{E}+00$ \\
\hline Sample Control & $4.08 \mathrm{E}-01$ & $4.89 \mathrm{E}-04$ & $1.29 \mathrm{E}-02$ & $3.30 \mathrm{E}-03$ & $1.40 \mathrm{E}-04$ & $1.22 \mathrm{E}-05$ & 4.25E-01 \\
\hline \multicolumn{8}{|c|}{ Talinum triangulare } \\
\hline Q. Sample & $1.43 \mathrm{E}+00$ & $4.89 \mathrm{E}-04$ & $4.51 \mathrm{E}-02$ & $3.85 \mathrm{E}-01$ & $1.40 \mathrm{E}-04$ & $1.91 \mathrm{E}-01$ & $2.05 \mathrm{E}+00$ \\
\hline Sample Control & $3.01 \mathrm{E}+00$ & 4.89E-04 & $8.80 \mathrm{E}-03$ & $5.54 \mathrm{E}-01$ & $1.40 \mathrm{E}-04$ & $1.22 \mathrm{E}-05$ & $3.57 \mathrm{E}+00$ \\
\hline \multicolumn{8}{|l|}{ Citrus sinensis } \\
\hline Q. Sample & $3.65 \mathrm{E}-01$ & 4.89E-04 & $2.24 \mathrm{E}-02$ & $1.29 \mathrm{E}-01$ & $2.97 \mathrm{E}+01$ & $3.17 \mathrm{E}-01$ & $3.05 \mathrm{E}+01$ \\
\hline Sample Control & $3.84 \mathrm{E}-01$ & 4.89E-04 & $1.21 \mathrm{E}-02$ & $3.86 \mathrm{E}-02$ & 4.19E-02 & $1.22 \mathrm{E}-05$ & 4.77E-01 \\
\hline \multicolumn{8}{|c|}{ Chrysophyllum albidun } \\
\hline Q. Sample & $3.98 \mathrm{E}-01$ & $4.89 \mathrm{E}-04$ & $3.49 \mathrm{E}-02$ & $1.73 \mathrm{E}-01$ & $1.40 \mathrm{E}-04$ & $4.58 \mathrm{E}-01$ & $1.06 \mathrm{E}+00$ \\
\hline Sample Control & $7.15 \mathrm{E}+00$ & 4.89E-04 & $1.73 \mathrm{E}-02$ & $5.05 \mathrm{E}-01$ & $1.40 \mathrm{E}-04$ & $1.22 \mathrm{E}-05$ & $7.67 \mathrm{E}+00$ \\
\hline \multicolumn{8}{|l|}{ Carica papaya } \\
\hline Q. Sample & $1.23 \mathrm{E}+00$ & 4.89E-04 & $2.57 \mathrm{E}-02$ & $8.89 \mathrm{E}-01$ & 4.05E-01 & $9.80 \mathrm{E}-01$ & $3.53 \mathrm{E}+00$ \\
\hline Sample Control & $6.98 \mathrm{E}-03$ & 4.89E-04 & $4.89 \mathrm{E}-03$ & $4.70 \mathrm{E}-03$ & $1.40 \mathrm{E}-04$ & $1.22 \mathrm{E}-05$ & $1.72 \mathrm{E}-02$ \\
\hline \multicolumn{8}{|l|}{ Cocos nucifera } \\
\hline Q. Sample & $1.86 \mathrm{E}+00$ & $4.89 \mathrm{E}-04$ & $1.21 \mathrm{E}-02$ & $1.92 \mathrm{E}-01$ & $1.40 \mathrm{E}-04$ & $1.22 \mathrm{E}-05$ & $2.06 \mathrm{E}+00$ \\
\hline Sample Control & $6.44 \mathrm{E}+00$ & 4.89E-04 & $1.29 \mathrm{E}-02$ & $4.54 \mathrm{E}-01$ & $1.40 \mathrm{E}-04$ & $1.22 \mathrm{E}-05$ & $6.91 \mathrm{E}+00$ \\
\hline
\end{tabular}


Onyedikachi et al. / Ovidius University Annals of Chemistry 29 (2018) 8 - 24

\begin{tabular}{|c|c|c|c|c|c|c|c|}
\hline \multicolumn{8}{|l|}{ Cola acuminata } \\
\hline Q. Sample & $5.16 \mathrm{E}+00$ & 4.89E-04 & $1.20 \mathrm{E}-02$ & 5.09E-01 & $1.40 \mathrm{E}-04$ & $1.22 \mathrm{E}-05$ & $5.68 \mathrm{E}+00$ \\
\hline Sample Control & $3.21 \mathrm{E}-01$ & 4.89E-04 & $1.50 \mathrm{E}-02$ & 3.39E-02 & $1.40 \mathrm{E}-04$ & $1.22 \mathrm{E}-05$ & $3.71 \mathrm{E}-01$ \\
\hline \multicolumn{8}{|c|}{ Elaies guineensis } \\
\hline Q. Sample & 8.69E-01 & 4.89E-04 & $1.03 \mathrm{E}-02$ & $1.95 \mathrm{E}-02$ & $1.40 \mathrm{E}-04$ & $1.22 \mathrm{E}-05$ & 8.99E-01 \\
\hline Sample Control & $2.83 \mathrm{E}-01$ & 4.89E-04 & $5.21 \mathrm{E}-03$ & $5.66 \mathrm{E}-02$ & $3.35 \mathrm{E}-01$ & $1.22 \mathrm{E}-05$ & $6.80 \mathrm{E}-01$ \\
\hline \multicolumn{8}{|l|}{ Average } \\
\hline Quarry site & $2.42 \mathrm{E}+00$ & $1.15 \mathrm{E}-02$ & 2.01E-02 & $4.66 \mathrm{E}-01$ & $2.53 \mathrm{E}+00$ & $1.62 \mathrm{E}-01$ & $5.60 \mathrm{E}+00$ \\
\hline Control site & $2.64 \mathrm{E}+00$ & $1.53 \mathrm{E}-02$ & $1.63 \mathrm{E}-02$ & $2.45 \mathrm{E}-01$ & $8.27 \mathrm{E}-02$ & $1.28 \mathrm{E}-05$ & $2.99 \mathrm{E}+00$ \\
\hline
\end{tabular}

Table 9. Target hazard quotient and-hazardous indexes of heavy metals in selected food crops for children who live around Quarry sites (Ishiagu) and agricultural area (Umudike)

\begin{tabular}{|c|c|c|c|c|c|c|c|}
\hline Heavy metals & Mn & $\mathbf{C d}$ & $\mathbf{Z n}$ & $\mathbf{F e}$ & $\mathbf{P b}$ & $\mathbf{C u}$ & HI \\
\hline \multicolumn{8}{|c|}{ Manihot esculenta } \\
\hline Q. Sample & $4.59 \mathrm{E}+00$ & $1.22 \mathrm{E}-01$ & $7.42 \mathrm{E}-03$ & $1.80 \mathrm{E}+00$ & $1.41 \mathrm{E}-01$ & $1.36 \mathrm{E}-05$ & $6.66 \mathrm{E}+00$ \\
\hline Control & $9.54 \mathrm{E}+00$ & 5.43E-04 & $1.10 \mathrm{E}-02$ & $1.02 \mathrm{E}+00$ & $1.55 \mathrm{E}-04$ & $1.36 \mathrm{E}-05$ & $1.06 \mathrm{E}+01$ \\
\hline \multicolumn{8}{|c|}{ Dioscorea rodundata } \\
\hline Q. Sample & $1.32 \mathrm{E}+00$ & $5.43 \mathrm{E}-04$ & $5.84 \mathrm{E}-03$ & $1.79 \mathrm{E}-01$ & $1.55 \mathrm{E}-04$ & $1.36 \mathrm{E}-05$ & $1.51 \mathrm{E}+00$ \\
\hline Control & $3.34 \mathrm{E}-01$ & $1.63 \mathrm{E}-01$ & $1.88 \mathrm{E}-02$ & $1.67 \mathrm{E}-02$ & $1.55 \mathrm{E}-04$ & $1.36 \mathrm{E}-05$ & $5.33 \mathrm{E}-01$ \\
\hline \multicolumn{8}{|l|}{ Colocacia a } \\
\hline Q. Sample & $6.88 \mathrm{E}+00$ & 5.43E-04 & $1.28 \mathrm{E}-02$ & $8.66 \mathrm{E}-01$ & $1.55 \mathrm{E}-04$ & $2.70 \mathrm{E}-05$ & $7.76 \mathrm{E}+00$ \\
\hline Control & $1.37 \mathrm{E}+00$ & 5.43E-04 & $5.09 \mathrm{E}-02$ & $2.44 \mathrm{E}-02$ & $1.55 \mathrm{E}-04$ & $2.70 \mathrm{E}-05$ & $1.45 \mathrm{E}+00$ \\
\hline \multicolumn{8}{|c|}{ Telferia Occidentalis } \\
\hline Q. Sample & $3.26 \mathrm{E}-01$ & $4.50 \mathrm{E}-04$ & $1.87 \mathrm{E}-02$ & $9.79 \mathrm{E}-02$ & $5.20 \mathrm{E}-02$ & $1.12 \mathrm{E}-05$ & 4.95E-01 \\
\hline Control & $1.28 \mathrm{E}+00$ & $4.50 \mathrm{E}-04$ & $1.66 \mathrm{E}-02$ & $1.20 \mathrm{E}-01$ & $5.65 \mathrm{E}-01$ & $1.12 \mathrm{E}-05$ & $1.98 \mathrm{E}+00$ \\
\hline \multicolumn{8}{|c|}{ Vernonia amygdalina } \\
\hline Q. Sample & $3.11 \mathrm{E}+00$ & $4.50 \mathrm{E}-04$ & $2.71 \mathrm{E}-02$ & $8.33 \mathrm{E}-02$ & $1.28 \mathrm{E}-04$ & $1.12 \mathrm{E}-05$ & $3.22 \mathrm{E}+00$ \\
\hline Control & $3.76 \mathrm{E}-01$ & $4.50 \mathrm{E}-04$ & $1.18 \mathrm{E}-02$ & $3.04 \mathrm{E}-03$ & $1.28 \mathrm{E}-04$ & $1.12 \mathrm{E}-05$ & 3.91E-01 \\
\hline \multicolumn{8}{|c|}{ Talinum triangulare } \\
\hline Q. Sample & $1.31 \mathrm{E}+00$ & $4.50 \mathrm{E}-04$ & $4.15 \mathrm{E}-02$ & $3.54 \mathrm{E}-01$ & $1.28 \mathrm{E}-04$ & $1.76 \mathrm{E}-01$ & $1.88 \mathrm{E}+00$ \\
\hline Control & $2.77 \mathrm{E}+00$ & $4.50 \mathrm{E}-04$ & $8.09 \mathrm{E}-03$ & $5.10 \mathrm{E}-01$ & $1.28 \mathrm{E}-04$ & $1.12 \mathrm{E}-05$ & $3.29 \mathrm{E}+00$ \\
\hline \multicolumn{8}{|l|}{ Citrus sinensis } \\
\hline Q. San & $3.36 \mathrm{E}-01$ & $4.50 \mathrm{E}-04$ & $2.06 \mathrm{E}-02$ & $1.19 \mathrm{E}-01$ & $2.73 \mathrm{E}+01$ & $2.92 \mathrm{E}-01$ & $2.81 \mathrm{E}+01$ \\
\hline Control & $3.53 \mathrm{E}-01$ & $4.50 \mathrm{E}-04$ & $1.11 \mathrm{E}-02$ & $3.55 \mathrm{E}-02$ & $3.85 \mathrm{E}-02$ & $1.12 \mathrm{E}-05$ & 4.39E-01 \\
\hline \multicolumn{8}{|c|}{ Chrysophyllum albidun } \\
\hline Q. Sample & $3.66 \mathrm{E}-01$ & $4.50 \mathrm{E}-04$ & $3.21 \mathrm{E}-02$ & $1.59 \mathrm{E}-01$ & $1.28 \mathrm{E}-04$ & $4.22 \mathrm{E}-01$ & $9.80 \mathrm{E}-01$ \\
\hline Control & $6.58 \mathrm{E}+00$ & $4.50 \mathrm{E}-04$ & $1.59 \mathrm{E}-02$ & $4.65 \mathrm{E}-01$ & $1.28 \mathrm{E}-04$ & $1.12 \mathrm{E}-05$ & $7.06 \mathrm{E}+00$ \\
\hline \multicolumn{8}{|l|}{ Carica papaya } \\
\hline Q. Sample & $8.00 \mathrm{E}-01$ & $4.50 \mathrm{E}-04$ & $2.37 \mathrm{E}-02$ & $8.18 \mathrm{E}-01$ & $3.73 \mathrm{E}-01$ & $9.02 \mathrm{E}-01$ & $2.92 \mathrm{E}+00$ \\
\hline Control & $2.60 \mathrm{E}-01$ & $4.50 \mathrm{E}-04$ & $4.50 \mathrm{E}-03$ & $4.33 \mathrm{E}-03$ & $1.28 \mathrm{E}-04$ & $1.12 \mathrm{E}-05$ & 2.69E-01 \\
\hline \multicolumn{8}{|l|}{ Cocos nucifera } \\
\hline Q. Sample & $1.72 \mathrm{E}+00$ & $4.50 \mathrm{E}-04$ & $1.11 \mathrm{E}-02$ & $1.77 \mathrm{E}-01$ & $1.28 \mathrm{E}-04$ & $1.12 \mathrm{E}-05$ & $1.91 \mathrm{E}+00$ \\
\hline Control & $5.93 \mathrm{E}+00$ & $4.50 \mathrm{E}-04$ & $1.18 \mathrm{E}-02$ & $4.18 \mathrm{E}-01$ & $1.28 \mathrm{E}-04$ & $1.12 \mathrm{E}-05$ & $6.36 \mathrm{E}+00$ \\
\hline \multicolumn{8}{|l|}{ Cola acuminata } \\
\hline Q. Sample & $4.75 \mathrm{E}+00$ & $4.50 \mathrm{E}-04$ & $1.10 \mathrm{E}-02$ & $4.68 \mathrm{E}-01$ & $1.28 \mathrm{E}-04$ & $1.12 \mathrm{E}-05$ & $5.23 \mathrm{E}+00$ \\
\hline Control & $2.96 \mathrm{E}-01$ & $4.50 \mathrm{E}-04$ & $1.38 \mathrm{E}-02$ & $3.12 \mathrm{E}-02$ & $1.28 \mathrm{E}-04$ & $1.12 \mathrm{E}-05$ & $3.42 \mathrm{E}-01$ \\
\hline
\end{tabular}


Onyedikachi et al. / Ovidius University Annals of Chemistry 29 (2018) 8 - 24

\begin{tabular}{|l|c|c|c|c|c|c|c|}
\hline Q. Sample & $1.13 \mathrm{E}+00$ & $4.50 \mathrm{E}-04$ & $9.52 \mathrm{E}-03$ & $1.80 \mathrm{E}-02$ & $1.28 \mathrm{E}-04$ & $1.12 \mathrm{E}-05$ & $1.16 \mathrm{E}+00$ \\
\hline Control & $6.42 \mathrm{E}-03$ & $4.50 \mathrm{E}-04$ & $4.80 \mathrm{E}-03$ & $5.21 \mathrm{E}-02$ & $3.08 \mathrm{E}-01$ & $1.12 \mathrm{E}-05$ & $3.72 \mathrm{E}-01$ \\
\hline Average & & & & & & & \\
\hline Quarry site & $2.22 \mathrm{E}+00$ & $1.06 \mathrm{E}-02$ & $1.84 \mathrm{E}-02$ & $4.28 \mathrm{E}-01$ & $2.32 \mathrm{E}+00$ & $1.49 \mathrm{E}-01$ & $5.15 \mathrm{E}+00$ \\
\hline Control site & $2.42 \mathrm{E}+00$ & $1.40 \mathrm{E}-02$ & $1.49 \mathrm{E}-02$ & $2.25 \mathrm{E}-01$ & $7.61 \mathrm{E}-02$ & $1.29 \mathrm{E}-05$ & $2.75 \mathrm{E}+00$ \\
\hline
\end{tabular}

Table 10. Carcinogenic risk of heavy metals in selected food crops from quarry site (Ishiagu) and agricultural area (Umudike)

\begin{tabular}{|c|c|c|c|c|c|c|c|c|c|c|c|c|}
\hline & & ADULT & & & & & & CHILDREN & & & & \\
\hline Heavy metals & $\mathrm{Mn}$ & $\mathrm{Cd}$ & $\mathrm{Zn}$ & $\mathrm{Fe}$ & $\mathrm{Pb}$ & $\mathrm{Cu}$ & $\mathrm{Mn}$ & $\mathrm{Cd}$ & $\mathrm{Zn}$ & $\mathrm{Fe}$ & $\mathrm{Pb}$ & $\mathrm{Cu}$ \\
\hline \multicolumn{13}{|c|}{ Manihot esculenta } \\
\hline Q. Sample & - & $5.06 \mathrm{E}-05$ & - & - & $4.58 \mathrm{E}-06$ & - & - & $4.64 \mathrm{E}-05$ & - & - & $4.20 \mathrm{E}-06$ & - \\
\hline Sample Control & - & $2.25 \mathrm{E}-07$ & - & - & \begin{tabular}{|l|}
$5.03 \mathrm{E}-09$ \\
\end{tabular} & - & - & $2.06 \mathrm{E}-07$ & - & - & $4.62 \mathrm{E}-09$ & - \\
\hline \multicolumn{13}{|c|}{ Dioscorea rodundata } \\
\hline Q. Sample & - & $2.25 \mathrm{E}-07$ & - & - & 5.03E-09 & - & - & $2.06 \mathrm{E}-07$ & - & - & $4.62 \mathrm{E}-09$ & - \\
\hline Sample Control & - & $6.75 \mathrm{E}-05$ & - & - & \begin{tabular}{|l|}
$5.03 \mathrm{E}-09$ \\
\end{tabular} & - & - & $6.19 \mathrm{E}-05$ & - & - & $4.62 \mathrm{E}-09$ & - \\
\hline \multicolumn{13}{|c|}{ Colocacia antiquorm } \\
\hline Q. Sample & - & $2.25 \mathrm{E}-07$ & - & - & $5.03 \mathrm{E}-09$ & - & - & $2.06 \mathrm{E}-07$ & - & - & $4.62 \mathrm{E}-09$ & - \\
\hline Sample Control & - & $2.25 \mathrm{E}-07$ & - & - & \begin{tabular}{|l|}
$5.03 \mathrm{E}-09$ \\
\end{tabular} & - & - & $2.06 \mathrm{E}-07$ & - & - & $4.62 \mathrm{E}-09$ & - \\
\hline \multicolumn{13}{|c|}{ Telferia Occidentalis } \\
\hline Q. Sample & - & $1.86 \mathrm{E}-07$ & - & - & $1.68 \mathrm{E}-06$ & - & - & $1.71 \mathrm{E}-07$ & - & - & $1.55 \mathrm{E}-06$ & - \\
\hline Sample Control & - & $1.86 \mathrm{E}-07$ & - & - & $1.83 \mathrm{E}-05$ & - & - & $1.71 \mathrm{E}-07$ & - & - & $1.68 \mathrm{E}-05$ & - \\
\hline \multicolumn{13}{|c|}{ Vernonia amygdalina } \\
\hline Q. Sample & - & $1.86 \mathrm{E}-07$ & - & - & $4.15 \mathrm{E}-09$ & - & - & $1.71 \mathrm{E}-07$ & - & - & $3.82 \mathrm{E}-09$ & - \\
\hline Sample Control & - & $1.86 \mathrm{E}-07$ & - & - & $4.15 \mathrm{E}-09$ & - & - & $1.71 \mathrm{E}-07$ & - & - & $3.82 \mathrm{E}-09$ & - \\
\hline \multicolumn{13}{|c|}{ Talinum triangulare } \\
\hline Q. Sample & - & $1.86 \mathrm{E}-07$ & - & - & 4.15E-09 & - & - & $1.71 \mathrm{E}-07$ & - & - & $3.82 \mathrm{E}-09$ & - \\
\hline Sample Control & - & $1.86 \mathrm{E}-07$ & - & - & \begin{tabular}{|l|}
$4.15 \mathrm{E}-09$ \\
\end{tabular} & - & - & $1.71 \mathrm{E}-07$ & - & - & $3.82 \mathrm{E}-09$ & - \\
\hline \multicolumn{13}{|l|}{ Citrus sinensis } \\
\hline Q. Sample & - & $1.86 \mathrm{E}-07$ & - & - & \begin{tabular}{|l|}
$8.83 \mathrm{E}-04$ \\
\end{tabular} & - & - & $1.71 \mathrm{E}-07$ & - & - & $8.13 \mathrm{E}-04$ & - \\
\hline Sample Control & - & $1.86 \mathrm{E}-07$ & - & - & $1.25 \mathrm{E}-06$ & - & - & $1.71 \mathrm{E}-07$ & - & - & $1.15 \mathrm{E}-06$ & - \\
\hline \multicolumn{13}{|c|}{ Chrysophyllum albidun } \\
\hline Q. Sample & - & $1.86 \mathrm{E}-07$ & - & - & $4.15 \mathrm{E}-09$ & - & - & $1.71 \mathrm{E}-07$ & - & - & $3.82 \mathrm{E}-09$ & - \\
\hline Sample Control & - & $1.86 \mathrm{E}-07$ & - & - & \begin{tabular}{|l|}
$4.15 \mathrm{E}-09$ \\
\end{tabular} & - & - & $1.71 \mathrm{E}-07$ & - & - & $3.82 \mathrm{E}-09$ & - \\
\hline \multicolumn{13}{|l|}{ Carica papaya } \\
\hline Q. Sample & - & $1.86 \mathrm{E}-07$ & - & - & $1.20 \mathrm{E}-05$ & - & - & $1.71 \mathrm{E}-07$ & - & - & $1.11 \mathrm{E}-05$ & - \\
\hline Sample Control & - & $1.86 \mathrm{E}-07$ & - & - & \begin{tabular}{|l}
$4.15 \mathrm{E}-09$ \\
\end{tabular} & - & - & $1.71 \mathrm{E}-07$ & - & - & $3.82 \mathrm{E}-09$ & - \\
\hline \multicolumn{13}{|l|}{ Cocos nucifera } \\
\hline Q. Sample & - & $1.86 \mathrm{E}-07$ & - & - & 4.15E-09 & - & - & $1.71 \mathrm{E}-07$ & - & - & $3.82 \mathrm{E}-09$ & - \\
\hline Sample Control & - & $1.86 \mathrm{E}-07$ & - & - & $4.15 \mathrm{E}-09$ & - & - & $1.71 \mathrm{E}-07$ & - & - & $3.82 \mathrm{E}-09$ & - \\
\hline \multicolumn{13}{|c|}{ Cola acuminata } \\
\hline Q. Sample & - & $1.86 \mathrm{E}-07$ & - & - & $4.15 \mathrm{E}-09$ & - & - & $1.71 \mathrm{E}-07$ & - & - & 3.82E-09 & - \\
\hline Sample Control & - & $1.86 \mathrm{E}-07$ & - & - & \begin{tabular}{|l|}
$4.15 \mathrm{E}-09$ \\
\end{tabular} & - & - & $1.71 \mathrm{E}-07$ & - & - & $3.82 \mathrm{E}-09$ & - \\
\hline \multicolumn{13}{|c|}{ Elaies guineensis } \\
\hline Q. Sample & - & $1.86 \mathrm{E}-07$ & - & - & \begin{tabular}{|l|}
$4.15 \mathrm{E}-09$ \\
\end{tabular} & - & - & $1.71 \mathrm{E}-07$ & - & - & 3.82E-09 & - \\
\hline Sample Control & - & $1.86 \mathrm{E}-07$ & - & - & 9.97E-06 & - & - & $1.71 \mathrm{E}-07$ & - & - & 9.17E-06 & - \\
\hline \multicolumn{13}{|l|}{ Average } \\
\hline Quarry site & & 4.39E-06 & & & \begin{tabular}{|l|}
$7.51 \mathrm{E}-05$ \\
\end{tabular} & & & $4.03 \mathrm{E}-06$ & & & $6.91 \mathrm{E}-05$ & \\
\hline Control site & & $5.80 \mathrm{E}-06$ & & & \begin{tabular}{|l|}
$2.46 \mathrm{E}-06$ \\
\end{tabular} & & & $5.32 \mathrm{E}-06$ & & & $2.26 \mathrm{E}-06$ & \\
\hline
\end{tabular}

\subsection{Discussion}

Heavy metal concentration in soils and selected food crops. Crop cultivation in contaminated soils results in absorption, translocation and subsequent bioaccumulation of trace metals to toxic levels in edible plant tissues. Human exposure to toxic heavy 
metals can be through the food chain. Thus, there is potential risk to human and animal health from consumption of harvested farm produce like vegetables, tubers, fruits and nuts grown on polluted soils. Although, $\mathrm{Fe}, \mathrm{Mn}, \mathrm{Zn}$, and $\mathrm{Cu}$, are essential trace elements in human diet, when present in excess they can produce deleterious systemic effects [6].

Results on Table 3 show the mean concentration of heavy metals ( $\mathrm{mg} / \mathrm{kg}$ dry weight) in soil and selected vegetables tubers, fruits and nuts from polluted (Ishiagu) and supposed unpolluted area (Umudike). Heavy metals analyzed in this study are as follows: lead $(\mathrm{Pb})$, cadmium $(\mathrm{Cd})$, copper $(\mathrm{Cu})$, manganese $(\mathrm{Mn})$, iron $(\mathrm{Fe})$ and zinc $(\mathrm{Zn})$. Statistical test of significance was done at $95 \%$ confidence level. The results showed that the relative abundance of heavy metals across the locations and all samples was in the order $\mathrm{Fe}>\mathrm{Mn}>\mathrm{Zn}>\mathrm{Pb}>\mathrm{Cu}>\mathrm{Cd}$. Also, for soils the order was $\mathrm{Fe}>\mathrm{Mn}>\mathrm{Zn}>\mathrm{Cu}>\mathrm{Pb}>\mathrm{Cd}$. Relative abundance of heavy metals in food samples from the industrialized area (Ishiagu) was in the order $\mathrm{Fe}>\mathrm{Mn}>\mathrm{Pb}>\mathrm{Cu}>\mathrm{Zn}>\mathrm{Cd}$. While the concentration of heavy metals in samples from the commercial and residential area (Umudike) was found to be $\mathrm{Fe}>\mathrm{Mn}>\mathrm{Zn}>\mathrm{Pb}>\mathrm{Cd}>\mathrm{Cu}$. Findings show that food crops from the industrialized area (Ishiagu) accumulated heavy metals in the order tubers $>$ fruits $>$ nuts $>$ vegetables, while those from the agricultural zone followed the order tubers > vegetables > fruits > nuts. Thus, root tubers accumulated more heavy metals compared to other food crops. This is in consonance with previous studies [23-28] and followed the general trend observed for trace metal accumulation in food crops [28]. Among heavy metals analyzed in this study, iron levels was highest in edibles and rhizosphere soils which could be as a results of its presence in the earth crust i.e., parent rock material. This justifies its use as reference metal in the computation of enrichment factor [29, 30].

A one way between subjects ANOVA (analysis of variance) was conducted to compare the concentration of heavy metals in test and control samples from Ishiagu and Umudike respectively. The means were treated as significantly different at $p<$ 0.05. Result shows that there was (statistically determined) significant effect of quarrying activities on the concentration of the six investigated heavy metals (Fe, Mn, $\mathrm{Zn}, \mathrm{Pb}, \mathrm{Cu}, \mathrm{Cd})$ at $p<0.05$ level.

Mean manganese concentration in food samples from Ishiagu was within the range of $10.16 \mathrm{mg} / \mathrm{kg}$ dry weight (dw) to $177.4 \mathrm{mg} / \mathrm{kg} \mathrm{dw}$ while those from Umudike was within the interval of $0.20 \mathrm{mg} / \mathrm{kg}$ dw to $245.90 \mathrm{mg} / \mathrm{kg} \mathrm{dw}$. About $58 \%$ and $50 \%$ of samples from Ishiagu and Umudike respectively were above the joint FAO/WHO permissible limits for $\mathrm{Mn}$ in edible plants [23]. Mean manganese concentration in rhizosphere soils increased from $65.55 \mathrm{mg} / \mathrm{kg}$ to $248.50 \mathrm{mg} / \mathrm{kg} \mathrm{dw}$ and $6.50 \mathrm{mg} / \mathrm{kg} \mathrm{dw}$ to 275.60 $\mathrm{mg} / \mathrm{kg} \mathrm{dw}$ for Ishiagu and Umudike respectively.
Mean concentration of $\mathrm{Mn}$ in soils from the areas under study were within the globally accepted range for $\mathrm{Mn}$ and this is in consonance with previous studies. For tubers, mean concentration of manganese is highest in cocoyam and cocoyam test soils but lowest in yam samples. Results show that mean manganese concentration is higher in soil samples compared to that of the edible vegetables. Also, bitter leaf sample and soil appear to have the highest concentration of manganese when compared with other vegetables. Nuts showed higher manganese concentration when compared to fruit samples. A one way between subject ANOVA indicated that there was significant effect of anthropogenic activities on mean manganese concentration between the researched areas $[\mathrm{F}(23,24)=1.19 \mathrm{E}+06 \mathrm{mg} / \mathrm{kg} \mathrm{dw}$, $p=0.00]$. Post HOC comparism using the turkey LSD test indicated a significant difference in manganese levels (at $p<0.05$ level) within test and control groups for vegetables, tubers, fruits and nuts. Also, statistical significant differences exist between contaminated samples and their respective controls. There was similar observation for test and control soils.

Mean cadmium concentration was below WHO detection limit of $0.0001 \mathrm{mg} / \mathrm{kg}$ for all test samples and controls except for cassava test sample $(0.23 \mathrm{mg})$ and yam control sample $(0.30 \mathrm{mg})$. Mean cadmium levels in top soil was below WHO detection limit of $0.0001 \mathrm{mg} / \mathrm{kg}$ for the two sampled areas. Adsorption of cadmium ions into surfaces of clay minerals, reduces its mobility under acidic or alkaline conditions. One way between subject ANOVA indicated that there was significant effect of human activities on mean cadmium level (at $p<0.05$ level) between the two areas under investigation $[\mathrm{F}(23,24)$ $=2.14 \mathrm{E}+02, p=0.00]$. Multiple comparism showed that there is no statistical significant difference in mean cadmium level (at $p<0.05$ level) within control group, within test group, and between both groups for edibles and soil. This is with exception to cassava sample gotten around quarry mines $(0.23 \pm 0.03 \mathrm{mg} / \mathrm{kg}$ dry weight) and yam control $(0.30 \pm 0.00 \mathrm{mg} / \mathrm{kg}$ dry weight) which varied significant from all other samples and was slightly above FAO/WHO permissible limit of $0.21 \mathrm{mg} / \mathrm{kg}$ cadmium concentration in edibles [27].

The mean concentration of $\mathrm{Zn}$ in the selected food crops increased from $3.23 \pm 0.03 \mathrm{mg} / \mathrm{kg}$ dry weight to $27.71 \pm 0.01 \mathrm{mg} / \mathrm{kg}$ dry weight in waterleaf from Ishiagu while samples from Umudike showed an increase from $3.00 \pm 0.00 \mathrm{mg} / \mathrm{kg}$ dry weight to $28.10 \pm 0.00 \mathrm{mg} / \mathrm{kg}$ dry weight in yam. These values were below the joint FAO/WHO allowable limit of $47.4 \mathrm{mg} / \mathrm{kg} \mathrm{Zn}$ levels in edibles. For tubers, mean zinc level was highest in cocoyam samples and soil. Mean zinc levels in rhizosphere soils increased from $2.7 \mathrm{mg}$ in cassava soil to $53.95 \mathrm{mg} / \mathrm{kg} \mathrm{dw}$ in cocoyam soil and $5.40 \mathrm{mg}$ in water leaf soil to $29.20 \mathrm{mg} / \mathrm{kg} \mathrm{dw}$ in yam soil for Ishiagu and Umudike respectively. Mean zinc 
levels in rhizosphere soils were below limits set by international regulatory bodies. Mean zinc concentration in edible vegetables and their respective soils appear to be very close for both test samples and controls. Suggesting that $\mathrm{Zn}$ levels in plants was mainly due to plant uptake. Also, vegetables had the uppermost zinc levels compared to other edibles with waterleaf at the peak. Fruits had higher amounts of zinc compared to nuts with star apple showing high levels when compared to other fruits and nuts. This is suggestive of the view that vegetables and fruits can serve as rich sources of $\mathrm{Zn}$ in the diets of the populace. One way between subject ANOVA indicated that there was significant effect of anthropogenic activities on mean zinc concentration between the researched areas $[\mathrm{F}(23,24)=7.36 \mathrm{E}+03$ $\mathrm{mg} / \mathrm{kg} \mathrm{dw}, p=0.00]$. LSD multiple comparism tables showed that there was statistical significant differences (at $p<0.05$ level) for food crops within the polluted group, within control group and when both groups were compared. This is with exception to coconut and kola nut test samples which showed significant differences $(p=0.67)$. Also, rhizosphere soils showed statistical significant difference in mean zinc concentration within and between groups with exception to polluted yam soil and its control soil that were statistically insignificant ( $p=0.822$ ).

Mean Fe concentration in food samples from the proposed polluted site decreased from 2,654.11 $\mathrm{mg} / \mathrm{kg} \mathrm{dw}$ in cassava to $31.95 \mathrm{mg} / \mathrm{kg}$ dry weight in palm nut while those from Umudike decreased from $7,148.45 \mathrm{mg} / \mathrm{kg} \mathrm{dw}$ in cocoyam to $148.00 \mathrm{mg}$ in bitter leaf. These values were extremely higher than the permissible limit of $20 \mathrm{mg} / \mathrm{kg}$ set by FAO/WHO for Fe in edible plants. Rhizosphere soil samples showed decrease in Fe content from 1,496.40 mg in cassava soil to $5.40 \mathrm{mg}$ in bitter leaf soil and $7,301.20 \mathrm{mg} / \mathrm{kg}$ $\mathrm{dw}$ in cocoyam soil to $899.31 \mathrm{mg}$ in pumpkin soil for Ishiagu and Umudike respectively. As at the time of this research, regulatory limit for $\mathrm{Fe}$ levels in soils was not available. Generally, iron levels in food and soil samples was highest compared to other metals. Iron levels was higher in tubers compared to vegetables, fruits and nuts with cassava $(2654.11 \pm 0.11 \mathrm{mg} / \mathrm{kg} \mathrm{dw})$ having highest amounts. Amid the selected vegetables, water leaf test sample and control had chief Fe content. Quarry and control cocoyam soils had highest mean iron levels when compared to other soil samples. The high amount of iron in edible plants and top soils attest to the fact that Fe originates mainly from soil parent rock materials thus, its use as reference metal for the determination of enrichment factors. One way between subject ANOVA shows that there was significant contribution of extraction activities on iron concentration in samples gotten from farms proximal to quarry sites $[\mathrm{F}(23,24)=5.62 \mathrm{E}+05 \mathrm{mg} / \mathrm{kg} \mathrm{dw}, p=$ 0.00]. Multiple comparism using the turkey LSD test indicated a statistically relevant discrepancy in mean iron levels (at $p<0.05$ level) within test and control groups for vegetables, tubers, fruits and nuts. Also, statistical significant differences exist between contaminated samples and their respective controls. These observations were consistent for test and control soils.

Mean lead concentration was below WHO detection limit (BDL) of $0.0001 \mathrm{mg} / \mathrm{kg}$ in tubers, nuts, fruits and vegetables with exception to pumpkin (4.40 $\mathrm{mg} / \mathrm{kg} \mathrm{dw}$ ) from the supposed control sites, orange $(212.66 \mathrm{mg} / \mathrm{kg} \mathrm{dw})$ and cassava $(0.91 \mathrm{mg} / \mathrm{kg} \mathrm{dw})$ both from the quarry site. Biochemical and physical processes involving $\mathrm{Pb}$ binding, inactivation and precipitation impede the translocation of $\mathrm{Pb}$ from absorbing roots, resulting in low $\mathrm{Pb}$ levels in plant parts [8]. Mean lead concentration in orange test sample was extremely high compared to all other samples. Elevated $\mathrm{Pb}$ levels in orange may reflect the physiology of the plant resulting in high affinity for $\mathrm{Pb}$ and suggesting its use in phytoremediation of $\mathrm{Pb}$ polluted sites. Mean lead concentration in pumpkin (4.40 mg/kg dw) from Umudike, orange $(212.66 \mathrm{mg}$ ) and cassava $(0.91 \mathrm{mg})$ both from Ishiagu were above the permissible limit of $0.43 \mathrm{mg}$ set by WHO and FAO for $\mathrm{Pb}$ in edible plants. $\mathrm{Pb}$ content of rhizosphere soils were below WHO detection limit of $0.0001 \mathrm{mg} / \mathrm{kg}$ for all test soils and controls with exception to yam soil from Ishiagu, cassava and pumpkin soils from Umudike. Mean lead concentration in soils from the areas under study were within the safe reference range for $\mathrm{Pb}$ in soils. One way between subject ANOVA pointed out a significant effect of quarrying activities on mean Lead levels between Ishiagu and Umudike $[F(23,24)$ $=3.43 \mathrm{E}+06 \mathrm{mg} / \mathrm{kg} \mathrm{dw}, p=0.00]$. LSD multiple comparism box reveals that there was negligible statistical difference in mean lead concentration (at $p$ $<0.05$ level) between test and control group with exceptions to polluted cassava, orange, pawpaw, palm nut, pumpkin and pumpkin soil which were statistically different compared to their respective controls. Within the test group, there was statistical significant difference in mean lead levels for the three fruits and vegetables with exceptions to bitter leaf and water leaf test samples which showed no statistically relevant difference. In contrast, mean lead concentration within the test group was statistically insignificant for the three nuts and tubers except for cassava from the polluted site. Statistical significant difference was observed within uncontaminated group for fruits, nuts and vegetables excluding star apple and pawpaw, bitter leaf and water leaf, kola nut and coconut controls. With exception to yam and cocoyam soils, no statistical significant difference was observed when mean lead concentration for contaminated soils were juxtaposed. Dissimilar observations were made for the supposed unpolluted soils with exception to yam and cocoyam soils. Mean lead levels in polluted soils varied from below detection limit of $0.000100 \mathrm{mg} / \mathrm{kg}$ dry weight to $9.80 \pm 0.2000 \mathrm{mg} / \mathrm{kg}$ dry weight, while control soils 
were between 0.0001 and $3.72 \pm 0.2000 \mathrm{mg} / \mathrm{kg}$ dry weight. Mean lead concentration ranged from below WHO detection limit of $0.0001 \mathrm{mg}$ to $212.66 \pm 0.06$ $\mathrm{mg} / \mathrm{kg}$ dry weight in samples from Ishiagu while those from the supposed control area Umudike ranged from below detection limit of $0.0001 \mathrm{mg}$ to $4.40 \pm 0.00$ $\mathrm{mg} / \mathrm{kg}$ dry weight. Thus lead concentration in samples from quarry site was far above WHO permissible limit of $0.30 \mathrm{mg} / \mathrm{kg}$ in edibles. The populace at Ishiagu are therefore at risk of $\mathrm{Pb}$ poisoning. This could be the result of geoaccumulation of lead from anthropogenic sources around rhizosphere soils in the polluted area. Bioaccumulation of this metal in plant (livestock and human) tissues is the consequence of plant uptake (consumption). Lead plays no physiological role in man or animal system and thus not required even at trace levels. Lead hampers many metabolic pathways and processes. $\mathrm{Pb}$ poisoning can disrupt the functioning of several tissues, organs and systems including the heart, kidneys, bones, intestine, nervous and reproductive systems [28]. It is particularly toxic to infants because of their smaller bodies and resulting in behavioral and learning disorders [31]. Symptoms of $\mathrm{Pb}$ intoxication include confusion, headache, anaemia, abdominal pain, irritability etc. With serious intoxication, coma, seizures, and death may result [32]. In the nearest future, a threatening situation of lead poisoning may occur in study area. With exception to fruits and waterleaf test sample, mean concentration of copper in edible samples from the researched areas was below detectable limits of $0.001 \mathrm{mg} / \mathrm{kg}$, suggesting that fruit may serve as rich sources of this trace metal. $\mathrm{Cu}$ concentration in topsoil from Ishiagu was between below detection limits and $80.21 \mathrm{mg}$ while soils from Umudike varied from below detection to $49.90 \mathrm{mg}$. Mean concentration of copper in three fruits and waterleaf from polluted site was above WHO permissible limits of $0.1 \mathrm{mg} / \mathrm{kg}$ dry weight of copper in edibles. Compared to other soils, waterleaf and cocoyam rhizosphere soils showed elevated copper levels. One way between subjects ANOVA indicated that there was a statistically significant effect of anthropogenic activities on mean copper levels between polluted and control samples. $[\mathrm{F}(23,24)=3.21 \mathrm{E}+05 \mathrm{mg} / \mathrm{kg} \mathrm{dw}, p$ $=0.00]$. Post HOC comparism using the turkey LSD test showed that the statistical difference in mean Copper levels (at $p<0.05$ level) within control group was trivial for vegetables, tubers, fruits and nuts. Three selected fruits (pawpaw, orange and star apple) and waterleaf from the polluted area showed statistical significant difference in mean copper levels when juxtaposed with all other samples both within the test group and against control group.

Bio-concentration factor. To express quantitatively the bioavailability and bioaccumulation patterns of these elements in edible plant tissues, the heavy metal accumulation potential of food samples was assessed and presented in Table 5. Total heavy metal extraction coefficient was higher in the proposed polluted site $($ Ishiagu $=1.57 \mathrm{E}+03)$ compared to the supposed control site (Umudike $=3.50 \mathrm{E}+02$ ) implicating once again quarrying activities as the source of the bio-accumulated heavy metals in plant tissues. The trend for bio-concentration factor or heavy metal extraction coefficient with regards to samples from Ishiagu is given as $\mathrm{Pb}>\mathrm{Cd}>\mathrm{Fe}>\mathrm{Zn}$ $>\mathrm{Cu}>\mathrm{Mn}$ while for Umudike the order is $\mathrm{Pb}>\mathrm{Cd}>$ $\mathrm{Fe}>\mathrm{Zn}>\mathrm{Cu}>\mathrm{Mn}$. Results show that plants had high accumulation potential for $\mathrm{Pb}$ and $\mathrm{Cd}$ which can be attributed to the high enrichment of these metals in soils and atmospheric deposition on shoot from nonferrous activities. The affinity coefficient for heavy metals in studied food samples from Ishiagu was in the order cassava $>$ pumpkin $>$ bitter leaf $>$ waterleaf $>$ cocoyam $>$ yam while those from Umudike followed the trend yam > waterleaf > cassava > pumpkin $>$ bitter leaf $>$ cocoyam. Results shows that cassava is a hyperaccumulator of the six researched metals, this can be attributed to the special physiology of the plant and the high enrichment of these metals in the polluted soils which are direct contact with cassava root tubers. Also, pumpkin from both polluted and control sites shows hyperaccumulation for $\mathrm{Pb}$ this can be attributed to high root uptake and atmospheric deposition on foliage. Compared to fruits and nuts, leafy vegetables easily take-up heavy metals and accumulate them in their edible tissues [33]. Heavy metals are easily accumulated in their edible tissues in amounts capable of causing health problems to humans and livestock [29].

All samples from polluted and control sites had high extraction coefficient for $\mathrm{Cd}$ particularly the tubers, this is expected since they are in direct contact with soil enriched with $\mathrm{Cd}$. Cadmium uptake may result in a wide range of lethal effects in plants. It can hamper several physiological processes in plants particularly, oxidative reactions and nitrogen metabolism [30]. Bitter leaf from polluted and control sites showed hyperaccumulation for $\mathrm{Zn}$ suggestive that bitter leaf can serve as a rich source of the metal. Bioaccumulation potencies of cassava for $\mathrm{Mn}$ are 1.80 and 9.28 for test samples and control respectively. The hyperaccumulation of $\mathrm{Mn}$ can perhaps be attributed to the significant physiological roles it plays in the plant. Currently, such plants having high affinity for their essential trace elements are used for phytoremediation. These findings suggest the use of cassava as a bio-indicator for environmental pollution.

Health risk assessment for Ishiagu and Umudike. Daily intake of heavy metals. Tables 6 and 7 show the estimated daily intake of $\mathrm{Fe}, \mathrm{Mn}, \mathrm{Zn}, \mathrm{Pb}, \mathrm{Cu}$ and $\mathrm{Cd}$ for adults and children. The daily intake of heavy metals (DIM) was found to depend on the concentration of heavy metals in food samples and amount in kilograms of the particular food eaten [30]. 
Based on average values, the result showed that daily intake of metals through food consumption in Ishiagu was in the ascending order $\mathrm{Cd}<\mathrm{Zn}<\mathrm{Cu}<\mathrm{Pb}<\mathrm{Mn}$ $<\mathrm{Fe}$ for both adults and children. In the control site (Umudike), the estimated daily intake of heavy metals (based on average values) varied by not less than multiples of $10^{\mathrm{n}}$ from the least $(\mathrm{Cu})$ to the highest $(\mathrm{Fe})$ and was in the order $\mathrm{Cu}<\mathrm{Cd}<\mathrm{Pb}<\mathrm{Zn}<\mathrm{Mn}$ $<\mathrm{Fe}$ for both adults and children. For samples from the quarry site, EDIM for $\mathrm{Pb}$ a known carcinogen was higher than some essential trace metals like $\mathrm{Cu}$ and $\mathrm{Zn}$ while known carcinogenic metals had among the least EDIM in the control region, indicating that quarrying activities in Ishiagu poses potential health and carcinogenic risk to the populace. The EDIM of all six heavy metals together was highest in cassava for the two sampled areas (for adults $1.64 \mathrm{mg} / \mathrm{kg} /$ day and $1.04 \mathrm{mg} / \mathrm{kg} /$ day while, calculated values for children was $1.51 \mathrm{mg} / \mathrm{kg} /$ day and $9.50 \mathrm{E}-01$ $\mathrm{mg} / \mathrm{kg} /$ day for test samples and controls respectively). The ingestion of heavy metals via food consumption was in the order tubers $>$ fruits $>$ nuts $>$ vegetables for Ishiagu while that of the control site was in order tubers $>$ vegetables $>$ fruits $>$ nuts. The estimated daily intake of heavy metals through vegetable consumption was highest in waterleaf $(3.49 \mathrm{E}-01$ $\mathrm{mg} / \mathrm{kg} /$ day and $4.88 \mathrm{E}-01 \mathrm{mg} / \mathrm{kg} /$ day) one of the most eaten vegetable in the researched areas. International regulatory bodies like the US EPA defined the reference oral dose (RfD) for heavy metals as an estimate of the tolerable daily intake of a metal by human population that will pose no health risk during a life time. It is usually employed for the assessment of EPA's non-carcinogenic health risk [14, 15]. RfD values for the six researched metals are presented in Table 1. According to international regulatory bodies, the provisional tolerable intake for $\mathrm{Cd}$ is $0.001 \mathrm{mg}$ $\mathrm{kg}^{-1} \mathrm{day}^{-1}$. This value is notably above the average daily intake for adults (of $1.15 \mathrm{E}-05 \mathrm{mg} \mathrm{kg}^{-1} \mathrm{day}^{-1}$ and $\left.1.53 \mathrm{E}-05 \mathrm{mg} \mathrm{kg}^{-1} \mathrm{day}^{-1}\right)$ and children (1.06 mg kg-1 day $^{-1}$ and $1.40 \mathrm{mg} \mathrm{kg}^{-1} \mathrm{day}^{-1}$ ) obtained in this study for Ishiagu and Umudike respectively. This suggests that the populace in the study area may not be ingesting toxic doses of $\mathrm{Cd}$. Average daily intake of $\mathrm{Zn}$ by adults through consumption of food crops was 6.02E-03 mg kg-1 day $^{-1}$ and 4.87E-03 mg kg-1 day $^{-1}$ while the average daily $\mathrm{Zn}$ intake by children was $5.54 \mathrm{E}-03 \mathrm{mg} \mathrm{kg}^{-1} \mathrm{day}^{-1}$ and $4.48 \mathrm{E}-03 \mathrm{mg} \mathrm{kg}^{-1}$ day $^{-1}$ for quarry and control site respectively. These values are below the oral dose of $0.3 \mathrm{mg} \mathrm{kg}^{-1} \mathrm{Zn} \mathrm{day}^{-1}$ recommended to be safe. $8.85 \mathrm{E}-03 \mathrm{mg} \mathrm{kg}^{-1} \mathrm{day}^{-1}$ and 2.89E-04 mg kg-1 $\mathrm{day}^{-1}$ (for adults) and $8.13 \mathrm{E}-03 \mathrm{mg}$ $\mathrm{kg}^{-1}$ day $^{-1}$ and $2.67 \mathrm{E}-04 \mathrm{mg} \mathrm{kg}^{-1}$ day $^{-1}$ are the daily oral exposures to $\mathrm{Pb}$ obtained for the people living around Ishiagu and Umudike respectively. These values are below the tolerable daily intake of 0.0035 $\mathrm{mg} \mathrm{kg}^{-1} \mathrm{day}^{-1}$ set by the US EPA. The reference oral dose for $\mathrm{Fe}$ is $0.8 \mathrm{mg} \mathrm{kg}^{-1} \mathrm{day}^{-1}$ as set by US EPA. The average estimated daily intake of $\mathrm{Fe}$ obtained in this study was $3.73 \mathrm{E}-01 \mathrm{mg} \mathrm{kg}^{-1} \mathrm{day}^{-1}$ and $1.95 \mathrm{E}-01$ $\mathrm{mg} \mathrm{kg}{ }^{-1} \mathrm{day}^{-1}$ (adults) also $3.43 \mathrm{E}-01 \mathrm{mg} \mathrm{kg}^{-1} \mathrm{day}^{-1}$ and $1.80 \mathrm{E}-01 \mathrm{mg} \mathrm{kg}^{-1} \mathrm{day}^{-1}$ (children) for the populace around quarry and control site respectively. These values are below the reference oral dose for $\mathrm{Fe}$ indicating that the human population within these locals may be ingesting safe levels of $\mathrm{Fe}$. On the average, daily $\mathrm{Cu}$ intake of $6.49 \mathrm{E}-03 \mathrm{mg} \mathrm{kg}^{-1}$ day $^{-1}$ and $5.14 \mathrm{E}-07 \mathrm{mg} \mathrm{kg}^{-1} \mathrm{day}^{-1}$ as obtained in this study for Ishiagu and Umudike suggest a safe oral dose since values are away below the recommended daily intake of $0.04 \mathrm{mg} \mathrm{kg}^{-1} \mathrm{Cu}$ per day as defined by regulatory bodies. The recommended daily intake by international regulatory bodies for $\mathrm{Mn}$ is $0.014 \mathrm{mg}$ $\mathrm{kg}^{-1} \mathrm{day}^{-1}$. Daily intake for $\mathrm{Mn}$ in investigated samples was between $4.96 \mathrm{E}-03 \mathrm{mg} \mathrm{kg}^{-1} \mathrm{day}^{-1}$ in pumpkin to $1.05 \mathrm{E}-01 \mathrm{mg} \mathrm{kg}^{-1} \mathrm{day}^{-1}$ in cocoyam with an average of $3.38 \mathrm{E}-02 \mathrm{mg} \mathrm{kg} \mathrm{day}^{-1}$ for the populace at Ishiagu. Average daily intake of $\mathrm{Mn}$ for Umudike was $3.70 \mathrm{E}-02 \mathrm{mg} \mathrm{kg}^{-1} \mathrm{day}^{-1}$. Average values are above the limits set by US EPA.

Target hazard quotient and hazardous indexes of heavy metal in selected food crops. In order to give an estimate of the non-carcinogenic health risk due to oral exposure to heavy metals, the target hazard quotient and hazardous indexes of heavy metal in selected food crops was calculated. Though, the THQ does not reveal the extent in (quantitative terms) to which an exposed population experiences health risk it does indicate levels of concern [14, 15]. Generally if THQ $<1$ there is no health risk associated with the current level of exposure to a given substance and the population under study is considered to be safe. Reverse is the case when THQ > 1 . In this study, Tables 8 and 9 presented the average THQ values obtained for $\mathrm{Mn}, \mathrm{Cd}, \mathrm{Zn}, \mathrm{Fe}, \mathrm{Pb}$ and $\mathrm{Cu}$ was 2.42E+00, 1.15E-02, 2.01E-02, 4.66E-01, 2.53E+00 and 1.62E-01respectively for samples from Ishiagu. Average target hazard quotients obtained for Mn and $\mathrm{Pb}$ was $>1$ indicating that the residents at the quarry site may be exposed to potential non-carcinogenic health risk due to $\mathrm{Mn}$ and $\mathrm{Pb}$ intoxication. Food samples from Umudike had average THQ values of $2.64 \mathrm{E}+00,1.53 \mathrm{E}-02,1.63 \mathrm{E}-02,2.45 \mathrm{E}-01,8.27 \mathrm{E}-$ 021.28E-05. Thus, $\mathrm{Mn}$ poses possible noncarcinogenic health risk for the populace at Umudike. For individual samples, the THQs of $\mathrm{Cd}, \mathrm{Zn}$ and $\mathrm{Cu}$ for food crops from Ishiagu and Umudike were less than one which implies that the daily intake of these metals by the populace were below the reference oral dose and the population is may be safe from $\mathrm{Cd}, \mathrm{Zn}$ and $\mathrm{Cu}$ intoxication. Considering samples individually, health risk indexes for $\mathrm{Fe}$ and $\mathrm{Pb}$ in all analyzed samples were also below one with exception to cassava from Ishiagu and orange from Umudike. Mn showed THQ values greater than one for about $67 \%$ of samples from Ishiagu and $50 \%$ of samples from Umudike. Krishna et al. [34] reported that maximum THQ value poses relatively higher potential health risk to human beings especially for residents in the locals with serious metal 
contamination. Thus, the daily intake of Mn for most of the samples from Ishiagu were above the reference oral dose indicating a level of concern for $\mathrm{Mn}$ intoxication in the research areas [34]. The cancer slope factors were used to estimate the carcinogenic risk (CR) and results are presented in Table 10 . Average predictable life time risk to cancer due to $\mathrm{Pb}$ intake estimated in this study was 7.51E-05 for Ishiagu and 2.46E-06 for Umudike. With regards to US EPA standard, these values fall within the range of predictable lifetime risk to cancer $[11,20]$. On the average, the lifetime probability of contracting cancer by ingesting $\mathrm{Cd}$ through food crops grown around quarry mines as obtained in this study was 4.39E-06 while carcinogenic risk value obtained for those ingesting $\mathrm{Cd}$ through food crops grown around Umudike was 5.80E-06. These values indicate a lifetime (70 years) probability of contracting cancer with respect to US EPA prescriptions. Average carcinogenic risk values obtained for $\mathrm{Pb}$ and $\mathrm{Cd}$ in this study, suggest that they be placed for further consideration as chemicals of concern [18]. As at the time of this research, there was inadequate human and animal data on the carcinogenicity or equivocal mutagenicity for $\mathrm{Mn}, \mathrm{Zn}, \mathrm{Cu}$ and $\mathrm{Fe}[19,20]$.

\section{Conclusions}

This study presents a typical model for the probabilistic health risk assessment due to heavy metals intake through consumption of food crops. Total heavy metal extraction coefficient for food crops from around quarry mines (Ishiagu) was higher compared to values obtained for the supposed control site (Umudike) implicating once again, quarrying activities as a major source of the bio-accumulated heavy metals in plant tissues. $\mathrm{Mn}$ and $\mathrm{Fe}$ concentration in food samples were generally above the permissible limits set by WHO and FAO. Relative to other samples, cassava and pumpkin from the areas under study had elevated levels of lead and cadmium which were above the permissible limits of 0.43 $\mathrm{mg} / \mathrm{kg}$ and $0.21 \mathrm{mg} / \mathrm{kg}$ set by WHO and FAO [27]. Similar observation was made for their rhizosphere soils. Zn levels in food crops and rhizosphere soils from both sites was generally below the permissible limit for $\mathrm{Zn}$ in edible plants and agricultural soils. Thus, the populace in the researched areas may not be predisposed to $\mathrm{Zn}$ intoxication. With exception to fruits and waterleaf test sample, mean concentration of copper in edible samples from the researched areas were below detection limits (BDL) of $0.001 \mathrm{mg} / \mathrm{kg}$. Results show that ingestion of heavy metals via food consumption in the researched areas is mainly through tubers which serve as the most important staple foods in these areas. The DIM of all six heavy metals together was highest in cassava for the two sampled areas $(1.64 \mathrm{E}+00 \mathrm{mg} / \mathrm{kg} /$ day and $1.04 \mathrm{E}+00$ $\mathrm{mg} / \mathrm{kg} /$ day for test samples and controls respectively). With few exceptions the THQs for $\mathrm{Cd}, \mathrm{Cu}, \mathrm{Fe}$ and $\mathrm{Zn}$ calculated in this study were less than one suggesting that the investigated human populations may be free from non-carcinogenic health risk due to $\mathrm{Cd}, \mathrm{Cu}, \mathrm{Fe}$ and $\mathrm{Zn}$ intoxication. Average target hazard quotients obtained for $\mathrm{Mn}$ and $\mathrm{Pb}$ were > 1 indicating that the residents at the quarry site may be exposed to potential non-carcinogenic health risk due to $\mathrm{Mn}$ and $\mathrm{Pb}$ intoxication. Observed THQ values due to $\mathrm{Mn}$ on the average, was > 1 indicating possible noncarcinogenic health risk for the populace at Umudike. On the average, the carcinogenic health risk to residents ingesting cadmium and lead through food crops grown around quarry mines as obtained in this study indicated a lifetime (70 years) probability of contracting cancer with respect to US EPA prescriptions. Findings suggest that cadmium and lead be placed for further consideration as chemicals of concern with regards to the human populations studied.

Recommendations. A better understanding of the correlation between exposure, individual susceptibility and health effects of environmental pollutants like heavy metals investigated in this study facilitates the development of preventive and ameliorative measures, resulting in a decline in cancer risk for an exposed population. Several ecological and health risk parameters estimated in this study gave higher values for Ishiagu compared to Umudike implicating quarry activities as a major source of environmental contaminants. However, this study does not suggest complete safety for the populace in Umudike but advocate for more biomonitoring and environmental remediation programs in both areas especially for Ishiagu. Although, values for the calculated cancer risk for most of the samples (individually) was within the safe range, average values suggests a level of concern for $\mathrm{Pb}$ and $\mathrm{Cd}$ thus pointing to the need for further bio-monitoring studies. A combination of environmental health risk assessment and human bio-monitoring studies may synergistically promote a better understanding of the bioaccessibility and bioavailability of heavy metals which is important for the determination of potential health risk to humans. Elevated levels of heavy metals in cassava samples as shown by the metal extraction coefficient calculated in this study suggest its use as a bio-indicator of ecological and health hazards. Plants like bitter leaf and cassava with high affinity for trace metals essential for plant and human physiology may be used as phyto-remediators of $\mathrm{Zn}$, $\mathrm{Fe}, \mathrm{Cu}$ and $\mathrm{Mn}$. Also, this study did not assess the possible carcinogenic risk due to other types of hazardous substances like toxic gases which may be part of industrial effluent of which the additive effect may increase THQ and push CR values beyond the permissible limits set by US EPA. Furthermore, oral exposure to contaminants should not be the only criterion for assessing health risk, other routes of human exposure should be considered including 
gaseous intake (inhalation) and dermal deposition. To curtail environmental pollution and the associated health risk, pollution alleviation strategies like sensitizing the masses, proper management of industrial effluent, enacting laws that guide the release of domestic and industrial runoff to the surrounding lands, industrial localization away from residential areas, microbial-remediation and the use of weeds with no known medicinal or dietary role(s) in the site of interest as phyto-remediators may suffice.

Mitigating health risk challenges for an exposed population will require the collaborative efforts of the masses, policy makers local and international regulatory bodies like WHO, FAO, NAFDAC, NDLEA etc.

Conflicts of interest: The authors declare no conflict of interest.

\section{References}

[1]. World Health Organization-International Program for Chemical Safety (WHO-IPCS), 2010. Harmonization Project Document No. 8. WHO human health risk assessment toolkit: Chemical hazards. Inter-Organization Program for the Sound Management of Chemicals (IOMC).

[2]. A. Mahmood, R.N. Malik, Human health risk assessment of heavy metals via consumption of contaminated vegetables collected from different irrigation sources in Lahore, Pakistan, Arabian Journal of Chemistry 7 (2014) 91-99.

[3]. E.H. Aloh, O.G. Aloh, F.C. Otuu, E.N. Shu, C.I. Maduka, I.S. Inya-Agha, C.O.E. Nwando, B.H. Tilako, C.C. Okechukwu, Occupational health hazards associated with continuous exposure to quarry activities among quarry workers in Ebonyi State, South East Geopolitical Zone, Nigeria, IOSR Journal of Environmental Science, Toxicology and Food Technology 11 (2017) 10-19.

[4]. K.U. Osuocha, E.C. Chukwu, E.A. Ugbogu, O.C. Atasie, C.E. Ogbonna, Effects of quarry mining activities on the nutritional composition of edible vegetables in Ishiagu, Ebonyi state, Nigeria, Journal of Experimental Biology and Agricultural Sciences 4 (2016) 467-474.

[5]. S. Khan, Q. Cao, Y.M. Zheng, Y. Z. Huang, Y. G. Zhu, Health risks of heavy metals in contaminated soils and food crops irrigated with wastewater in Beijing, China, Environmental Pollution 152 (2008) 686-692.

[6]. P.F. Avila, S.E. Ferreira, C. Candeias, Health risk assessment through consumption of vegetables rich in heavy metals: the case study of the surrounding villages from Panasqueira mine, Central Portugal, Environmental Geochemistry and Health 39 (2017) 565-589.
[7]. R.C. Tasrina, A. Rowshon, A.M.R. Mustafzur, I. Rafqul, M.P. Ali, Heavy metals contamination in vegetables and its growing soil, Journal of Environmental Analytical Chemistry 2 (2015) 2-6.

[8]. M. Rezvan, M. Zaefarian, Bioaccumulation and translocation factors of cadmium and lead in Aeluropus littoralis, Australian Journal of Agricultural Engineering 2 (2011) 114-119

[9]. J.K. Nduka, O.E. Orisakwe, Assessment of environmental distribution of lead in some municipalities of South-Eastern Nigeria, International Journal of Environmental Research and Public Health 7 (2010) 25012513.

[10]. O.J. Ayodele, O.S. Shittu, T. Balogun, Heavy metal pollution assessment of granite quarrying operations at Ikole-Ekiti, Nigeria, International Journal of Environmental Monitoring and Analysis 2 (2014) 333-339.

[11]. K.W. Nkpaa, K.C.P. Iwuanyanwu. M.O. Wegwu, E. B Essien, Health risk assessment of hazardous metals for population via consumption of seafood from Ogoniland, Rivers State, Nigeria; A case study of Kaa, B-Dere, and Bodo City, Environmental Monitoring and Assessment 188 (2016) 1-10.

[12]. E.O. Nwaichi, M.O. Wegwu, U.L. Nwosu, Distribution of selected carcinogenic hydrocarbon and heavy metals in an oil-polluted agriculture zone, Environmental Monitoring and Assessment 186 (2014) 8697-8706.

[13]. X. Wang, T. Sato, X. Baoshan, Health risk of heavy metals to the general public of Tianjin, China via consumption of vegetables and fish, Science of the Total Environment 350 (2005) 2837.

[14]. F. Guerra, A.R. Trevizam, T. Muraoka, N.C. Marcante, Heavy metals in vegetables and potential risk for human health, Science and Agriculture 69 (2012) 54-60.

[15]. M.A.M. Mahmoud, H.S. Abdel-Mohsein, Health risk assessment of heavy metals for Egyptian population via consumption of poultry edibles, Advances in Animal and Veterinary Sciences 3 (2015) 58-70.

[16]. US EPA, Hubson River PCBs reassessment RI/FS Phase 2 Human Health Risk Assessment, (1998). Scope of work, Region II, U.S. Environmental Protection Agency, Washington, D.C. 290 Broadway New York. N.Y. 10007.

[17]. ATSDR, Agency for toxic substance and disease registry (2010). Public health assessment and health consultation. CENEX supply and marketing, Incorporated, Quicy, Grant County, Washington.

[18]. C.W. Liu, C.P. Liang, F.M. Huang, Assessing the human health risks from exposure of inorganic arsenic through oyster (Crassostrea 
gigas) consumption in Taiwan, Science of the Total Environment 361 (2007) 57-66.

[19]. US EPA. US EPA Regional Screening Level (RSL): Summary Table. Available at: http://www.epa.gov/regshwmd/risk/human/Ind ex.htm last update

[20]. US EPA. Screening level (RSL) for chemical contaminant at superfound sites, U.S. Environmental Protection Agency.

[21]. ECDGE, European Commission Director General Environment, Heavy Metals and Organic Compounds from Wastes Used as Organic Fertilizers (2010): Final Rep, July. Ref. Nr. TEND/AML/2001/07/20, pp. 73 - 74 http://ec.europa.eu/environment/waste/compost /pdf/hm_finalreport.pdf

[22]. S. Jabeen, M.T. Shah, S. Khan, M.Q. Hayat, Determination of major and trace elements in ten important folk therapeutic plants of Haripur basin, Pakistan, Journal of Medicinal Plants Research 4 (2010) 559-566.

[23]. O.O. Okedeyi, S. Dube, O. R. Awofolu, M. M. Nindi, Assessing the enrichment of heavy metals in surface soil and plant (Digitaria eriantha) around coal-fired power plants in South Africa, Environmental Science Pollution Research 21 (2014) 4686-4696.

[24]. G. Nabulo, O.H. Oryem, G.W. Nasinyama, G.D. Cole, Assessment of $\mathrm{Zn}, \mathrm{Cu}, \mathrm{Pb}$ and $\mathrm{Ni}$ contamination in wetland soils and plants in the Lake Victoria basin, International Journal of Environmental Science and Technology 5 (2008) 65-74.

[25]. F.O. Agunbiade, A.T. Fawale, Use of Siam weed biomarker in assessing heavy metal contamination in traffic and solid waste polluted areas, International Journal of Environmental Science and Technology 6 (2009) 267-276.

[26]. H. Wei, Z. Le, L. Shuxian, W. Dan, L. Xiaojun, J. Lan, M. Xiping, Health risk assessment of heavy metals and polycyclic aromatic hydrocarbons in soil at coke oven gas plants,
Environmental Engineering and Management Journal 14 (2015) 487-496.

[27]. FAO/WHO. Codex Alimentarius Commission. Food additives and contaminants. Joint FAO/WHO Food Standards Programme, 2001, pp. 1-289.

[28]. A.D. Woolf, R. Goldman, D.C. Bellinger, Update on the clinical management of childhood lead poisoning, Pediatric Clinics of North America 54 (2007) 271-94.

[29]. M.G. Alam, E.T. Snow, A. Tanaka, Arsenic and heavy metal contamination of vegetables grown in Samta village, Bangladesh, Science of Total Environment 308 (2003) 83-96.

[30]. H. Attila, S. Erdei, G. Horvath, Comparative studies of $\mathrm{H}_{2} \mathrm{O}_{2}$ detoxifying enzymes in green and greening barley seedlings under cadmium stress, Plant Science 160 (2001) 1085- 1093.

[31]. P.J. Landrigan, C.B. Schechter, J.M. Lipton, M.C. Fahs, J. Schwartz, Environmental pollutants and disease in American children, Environmental Health Perspectives 110 (2002) 721-728.

[32]. E.O. Nwaichi, M.O. Wegwu, U.L. Nwosu, Distribution of selected carcinogenic hydrocarbon and heavy metals in an oil-polluted agriculture zone, Environmental Monitoring and Assessment 186 (2014) 8697-8706.

[33]. F. Mapanda, E.N. Mangwayana, J. Nyamangara, K.E., Giller, Impacts of sewage irrigation on heavy metals distribution and contamination, Environmental International 31 (2005) 5-812.

[34]. P.V. Krishna, V. Jyothirmayi, K.M. Rao, Human health risk assessment of heavy metal accumulation through fish consumption, from Machilipatnam Coast, Andhra Pradesh, India, International Research Journal of Public and Environmental Health 1 (2014) 121-125.

Received: 27.032018

Received in revised form: 04.05.2018

Accepted: 07.05.2018 